\title{
Type-I and Type-II Core-Shell Quantum Dots: Synthesis and Characterization
}

Dirk Dorfs, Stephen Hickey and Alexander Eychmüller

\section{1 \\ Introduction}

The physical and optical properties of semiconductor quantum dots (QDs) are interesting for a variety of applications. However, due to their very large surface/ volume ratio, semiconductor QDs can also lose some of their desirable properties due to, for example, corrupted surfaces. Thus, the protection and passivation of the QD surface is of major importance for almost all of their possible applications. In most cases, this passivation is performed through the coating of a given semiconductor material with another material having a larger bulk bandgap than the core substance and a relative position of the bands, such that both charge carriers, the electrons, and the holes are confined to the core. This energetic situation is called type-I. In contrast, in type-II structures, the electrons and holes are separated so that one is located in the core material the other in the shell material. Both systems have been synthesized via various routes in recent years, and will be reviewed in the first part of the chapter. Subsequently, data will be provided relating to spherical semiconductor nanoheterostructures exhibiting more than one shell. The section describing ultraviolet-visible (UV-Vis) active nanomaterials concludes with a review on nonspherical core-shell systems, namely elongated rod-like structures, and a brief outline on the characterization of the aforementioned different nanoheterostructures. The timely consideration of steady developments presently being made in the area of infrared (IR) active materials is also addressed. Hence, a brief synopsis of the progress that has been achieved in the field to date is followed by a discussion of the synthesis and characterization of a number of near-infrared (NIR) nanomaterials is presented. Finally, a more indepth discussion of the resultant type-I and type-II core-shell NIR materials is undertaken. 


\section{2}

\section{Core-Shell UV-Vis Nanoparticulate Materials}

9.2.1

\section{Type-I Core-Shell Structures}

One of the main differences of semiconductor QDs in comparison with the corresponding bulk materials is the strongly increased band gap fluorescence observed in QDs. It could be shown that the quantum yield of a QD depends mainly on the surface properties of the nanocrystal. The quantum yield is high if the surface is well passivated, which means that all surface valences are saturated, for example, by organic ligands which are present in most QD systems as a consequence of the synthetic process.

However, these ligands may possibly be weakly bound, resulting in a dynamic equilibrium of adsorbing and desorbing ligands, and they can also be sensitive to all types of post-synthetic treatments of the QDs. Thus, a more stable method to saturate-and thus passivate-the nanoparticle surface was required in order to obtain QDs with high quantum yields and which also retained their fluorescence properties under various post-synthetic conditions.

The so-called core-shell structures were synthesized in order to passivate these surface traps, and thus increase the quantum efficiencies of the QD. Pioneering studies were conducted by Spanhel et al., who reported a significant enhancement of the band gap fluorescence of CdS QDs synthesized in water when treated with $\mathrm{OH}^{-}$in the presence of excessive $\mathrm{Cd}^{2+}[1]$. This increase in quantum yield was attributed to a passivating shell of $\mathrm{Cd}(\mathrm{OH})_{2}$ which formed around the QD and effectively hindered the free charge carriers from reaching the particle surface (and hence the trap states located at the particle surface).

Nowadays, most core-shell QDs are synthesized in high-boiling organic solvents based on the $\mathrm{CdE}(\mathrm{E}=\mathrm{S}, \mathrm{Se}, \mathrm{Te})$ synthesis of Murray et al. [2]. This synthesis yields samples with a much lower degree of polydispersity than the older water-based synthesis. The first example of a successful shell growth of another semiconductor material (ZnS in this case) onto CdSe QDs synthesized in organic solvents was reports by Guyot-Sionnest et al. in 1996 [3], and another very detailed study on the same system followed soon after by Bawendi et al. [4], while a systematic study of the growth of CdS onto CdSe QDs was reported by Peng et al. [5].

Both systems-CdSe/ZnS and CdSe/CdS core-shell nanocrystals-were subsequently studied in great detail, and are today standard systems when QDs with a high fluorescence quantum yield are required. The choice of $\mathrm{CdSe}$ as one of the most popular core materials for core-shell structures originates from its bulk band gap of $1.7 \mathrm{eV}$ (corresponding to a photon wavelength of $728 \mathrm{~nm}$ ), which allows the covering of almost the entire visible range of the spectrum with this material using QDs in a size regime of $1.7 \mathrm{~nm}$ to $6 \mathrm{~nm}$ in diameter. The choice of $\mathrm{ZnS}$ and CdS as popular shell materials for CdSe QDs originates from two different physical properties. While $\mathrm{ZnS}$ exhibits a wide band gap of $3.7 \mathrm{eV}$, which allows the free charge carriers to be effectively confined to the core of the core-shell particles even 

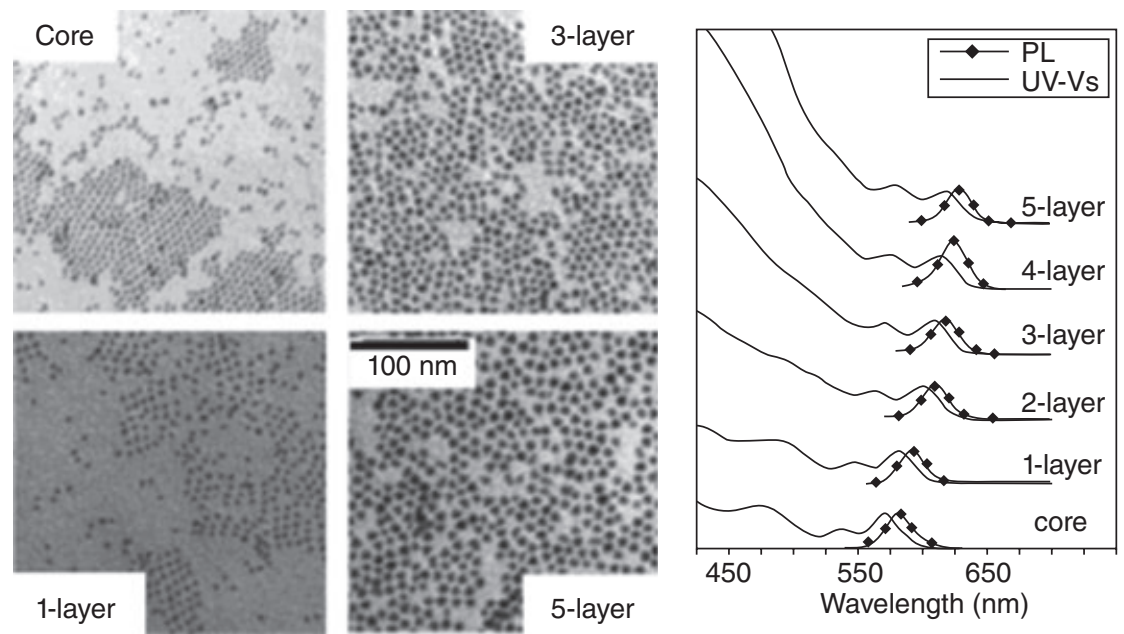

Figure 9.1 Left: Transmission electron microscopy images of $\mathrm{CdSe}$ nanocrystals and CdSe/CdS core-shell particles with one, three, and five ionic monolayers of a CdS shell; Right: The corresponding absorption and emission spectra [6].

with quite thin shells, it has a significant lattice mismatch with CdSe, which makes an epitaxial growth more challenging than for materials with a smaller lattice mismatch. CdS as a shell material for CdSe nanocrystals has a comparatively low lattice mismatch, allowing the epitaxial growth of thicker shells; however, the band gap of this material $(2.5 \mathrm{eV})$ is smaller than that of $\mathrm{ZnS}$, which causes the electronic passivation to be less effective at a given shell thickness.

Absorption and emission spectra of CdSe/CdS core-shell structures are shown in Figure 9.1. The main findings from these spectra are: (i) the first absorption maximum is shifted towards a lower energy with an increase in CdS shell thickness; and (ii) the fluorescence quantum yield is greatly enhanced in the core-shell QDs compared to the pure CdSe QDs. These findings can be explained as follows: the shift towards lower energies originates from a relaxation of the wavefunctions of both charge carriers into the CdS shell (however, the contribution of the electron should be much larger than that of the hole, as its effective mass is smaller and the band offsets between the conduction bands of both materials is smaller than the offset in the valance bands, both of which allow the electron to tunnel "more easily" into the shell than the hole), while the increase in quantum efficiencies originates from the passivation of surface trap states of the original QD and the much lower amplitude of the probability density function at the surface of the core-shell particle. For ZnS coating, which is the other very common coating material for CdSe, the observations are similar but also show two main differences: (i) the shift of the first absorption maximum towards lower energies is less pronounced; and (ii) the fluorescence quantum yield passes through a maximum at a thickness of the ZnS coating of 1.8 monolayers [4], while thicker coatings will 
cause the quantum yield to drop again. The less-pronounced shift of the first electronic transition can be explained with the very high band gap of ZnS compared to the band gap of CdS - hence, the relaxation of the charge carrier wavefunctions into the $\mathrm{ZnS}$ shell is much weaker than in the CdS case. The maximum in the fluorescence quantum yield can be explained by the large lattice mismatch between CdSe and ZnS, which causes considerable lattice strain and thus can cause crystallographic defects upon thicker ZnS coating.

The experimental procedures available for the colloidal synthesis of core-shell structures have improved considerably during the past decade. The first attempts dealt with aqueous media, and yielded materials with comparably high polydispersities. Subsequently, the pioneering studies of Guyot-Sionnest demonstrated the possibility of synthesizing core-shell structures in high-boiling organic solvents, and these showed a considerably lower polydispersity than their aqueous counterparts. The synthesis in high-boiling solvents was then further developed, for example, by Peng et al. [6], and this gave rise to an easy control of the shell thickness. Today, a variety of type-I core-shell structures can be synthesized in organic solvents, including CdSe/CdS [5], CdSe/ZnS [3, 4], CdS/ZnS [7], and InP/ZnS [8].

Due to their high fluorescence quantum yields and high photo- and chemical stabilities, type-I core-shell structures represent extremely interesting alternatives to common organic dye molecules with regards to labeling processes (e.g., "biolabeling," which is the in vitro or in vivo labeling of a bio molecule of interest which is then traced by detecting the photoluminescence (PL) of the QD [9]). Compared to the organic dyes in particular, the strongly enhanced photostability is important as well as the much narrower line width of the emission lines, which enables multicolor labeling.

\section{2 .2}

\section{Type-II Core-Shell Structures}

Whilst in type-I semiconductor heterostructures, the band gap alignment leads to a confinement of the charge carriers in one compartment of the heterostructure, the opposite is true for type-II nanoheterostructures.

The electron and hole are localized in two different compartments of the heterostructure in a type-II structure, and this usually leads to a significantly slowed down recombination rate of the charge carriers and thus to an extended luminescence lifetime.

While type-I nanoheterostructures are usually superior compared to homogeneous QDs in terms of their fluorescence quantum yield and photostability, type-II heterostructures have other major differences compared to type-I structures and homogeneous QDs. First, the staggered band alignment gives rise to a spatially indirect transition which occurs at lower energies than both of the band gaps of the two materials used (when not taking into account the additional quantization energy). Hence, type-II core-shell QDs can be emitters at wavelengths that cannot be achieved with any of the two materials alone. Furthermore, the luminescence lifetime of type-II heterostructures is strongly increased. 
Another, recently much-discussed, application of type-II core-shell QDs is the lasing of nanoparticles. Due to the type-II band alignment and lower overlap of the charge carrier wave functions, the exciton-exciton interaction becomes repulsive in type-II structures whilst being attractive in type-I systems. As demonstrated by Klimov et al., this behavior may prove advantageous when using QDs for amplified stimulated emission (ASE), which might open the way towards QD-based lasers.

Among the first colloidal type-II systems to be studied were CdTe/CdSe coreshell particles [10]. In these structures, the band offsets of the two materials cause the electron to be located in the CdSe shell, while the hole is confined to the core of the nanocrystals. The possibility of a spatially indirect transition from the CdTe valence band to the CdSe conduction band causes the absorption and emission wavelength of these structures to be strongly shifted towards lower energies compared to the pure CdTe nanoparticles, and even makes accessible wavelengths which would not be accessible with one of the two materials alone. Thus, the emission wavelength in these core-shell structures can be tuned up to $1000 \mathrm{~nm}$.

Another system that was intensively studied, especially in the context of ASE from nanoparticles, was the CdS/ZnSe core-shell nanoparticle [11], with the group of Klimov et al. successfully demonstrating their use for ASE. Figure 9.2 shows the emission spectra of CdS/ZnSe core-shell nanocrystals with two different CdS core sizes (1.6 and $2.4 \mathrm{~nm})$ and different ZnSe shell thicknesses [12]. The first finding here is that the emission wavelengths are smaller than the bulk band gaps of both materials $(\mathrm{CdS}=2.5 \mathrm{eV} ; \mathrm{ZnSe}=2.7 \mathrm{eV})$, and thus can be assigned to a

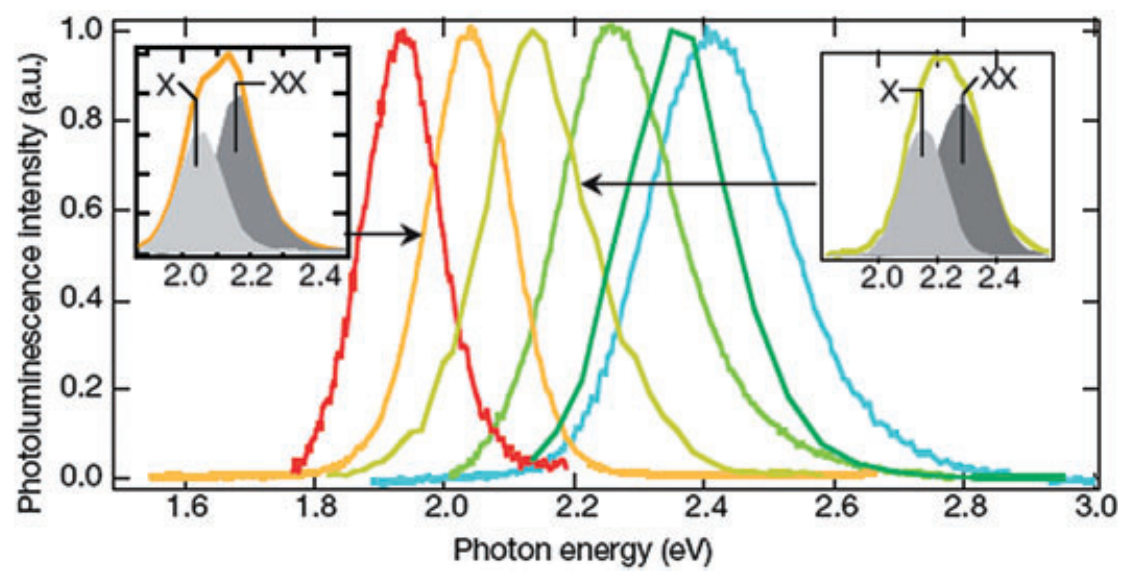

Figure 9.2 Emission spectra of CdS/ZnSe type-II core-shell nanocrystals (different core diameters and different shell thicknesses). The insets show emission spectra measured immediately after excitation at high pump intensities, which can be deconvoluted into a long-living part of the single excited particles and a very fast decaying part from doubleexcited particles [12]. 
spatially indirect recombination of a hole in the ZnSe valence band with an electron in the CdS conduction band (in the bulk, this transition would correspond to an energy of $1.8 \mathrm{eV}$ ).

The insets in Figure 9.2 show early time emission spectra measured immediately after the excitation at high pump energies. The main finding here is that the early time emission spectra can be deconvoluted into two signals, which can be assigned to a very fast decaying component originating from doublly excited particles (labeled as XX) and to a long-living component (labeled as X). The shift of the $\mathrm{XX}$ transition compared to the $\mathrm{X}$ transition has a magnitude of approximately $100 \mathrm{meV}$, and the XX transition is shifted towards higher energies. Both findings are related to the type-II structure of the nanocrystals. In type-I nanocrystals where the electron(s) and hole(s) are located in the same area of the nanocrystal, the exciton-exciton interactions are usually weak and attractive; thus, the $\mathrm{XX}$ transition would be shifted by small value towards lower energies. However, a different behavior is observed for type-II systems as, due to the spatial separation of electron(s) and hole(s), the exciton-exciton interaction becomes strong and repulsive, causing a much larger shift of the XX transition towards higher energies.

This behavior has interestingly an advantageous consequence when examining the ASE behavior of the nanocrystals. As can be seen in Figure 9.3 (left), ASE in type-I systems can only be observed at the wavelength of the XX transition, and hence from doubly excited particles. In the case of the type-II systems, however, ASE can be observed also from singly excited particles, and thus at the center of the ensemble emission wavelength. This behavior causes the pump energy threshold for ASE to be much lower in case of type-II systems than with type-I systems (see Figure 9.3, right). This behavior is responsible for type-II nanoheterostructures being very promising candidates for QD (rod)-based lasers.
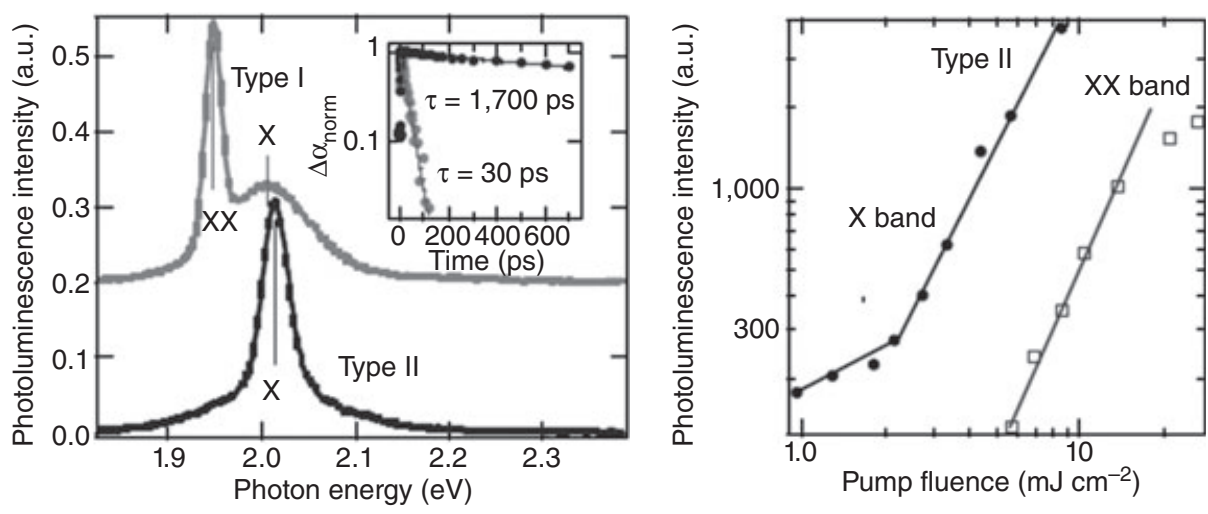

Figure 9.3 Left: ASE spectra of type-I nanocrystals (CdSe nanocrystals) and of type-II nanocrystals (CdS/ZnSe core-shell nanocrystals); Right: ASE intensity as a function of pump energy for type-I and type-II systems [12]. 


\section{2 .3}

\section{Multiple Shell Structures}

A more complicated structure, namely the QD quantum well system, which consists of CdS particles with an embedded layer of $\mathrm{HgS}$, was first synthesized in 1993 [13]. These particles were examined with different characterization techniques, including static and time-resolved photoluminescence, transient photobleaching, and high-resolution electron microscopy. The results obtained were compared with theoretical data, and the findings on this model system are summarized.

Likewise, research progress into different multishell nanocrystals will also be surveyed, describing three types of nanostructure: (i) Ternary core-shell shell systems with an intermediate layer as a "lattice adapter"; (ii) double QD quantum well (double-QDQW) systems; and (iii) an "inversed QDQW" system. Sorting multilayered nanocrystals into these three categories is justified by the different potential steppings of the semiconductor materials involved. Figure 9.4 shows the principal potential steppings of the valence and conduction bands in these three types of multilayered system.

The potential stepping on the left in Figure 9.4 causes both charge carriers (electron and hole) to be confined in the core of the nanocrystal (as above: type-I situation). In the case of QDQW systems (middle), the electron and hole are confined in the potential well, which is embedded in the QD. In the case of the "inversed" QDQW systems (right), the charge carriers are located in the core of the nanocrystal and in the outer shell. Depending on the different potential steppings, each of these systems will show unique properties (see below).

Based on polyphosphate-stabilized water-soluble CdS nanocrystals, the QDQW system consisting of CdS nanocrystals with an embedded layer of $\mathrm{HgS}$ (a QW within a QD) became a model system for a number of fundamental studies.

The synthetic concept was developed by Mews et al. in 1993 [13, 14]. To a solution of CdS QDs, $\mathrm{Hg}^{2+}$-ions were added; this resulted in a substitution reaction on the particle surface, where the outermost layer of Cd-ions was replaced by Hg-ions and the Cd-ions were released into the solution (cf. Figure 9.5). By analyzing the concentration of the free Cd- and Hg-ions in solution it was shown that, for an excessive addition of Hg-ions, no further substitution reaction took place, as no

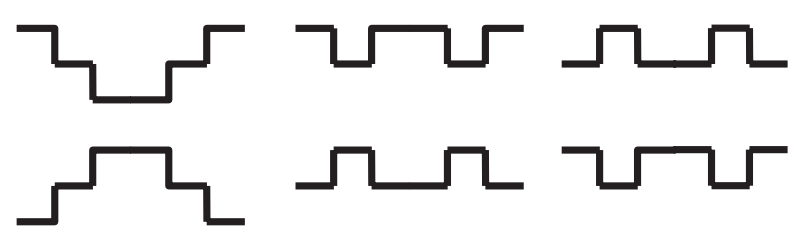

Figure 9.4 Potential stepping in core-shell-shell (CSS) nanocrystals (left), quantum dot quantum well (QDQW) nanocrystals (middle) and "inverse" QDQW nanocrystals (right). 

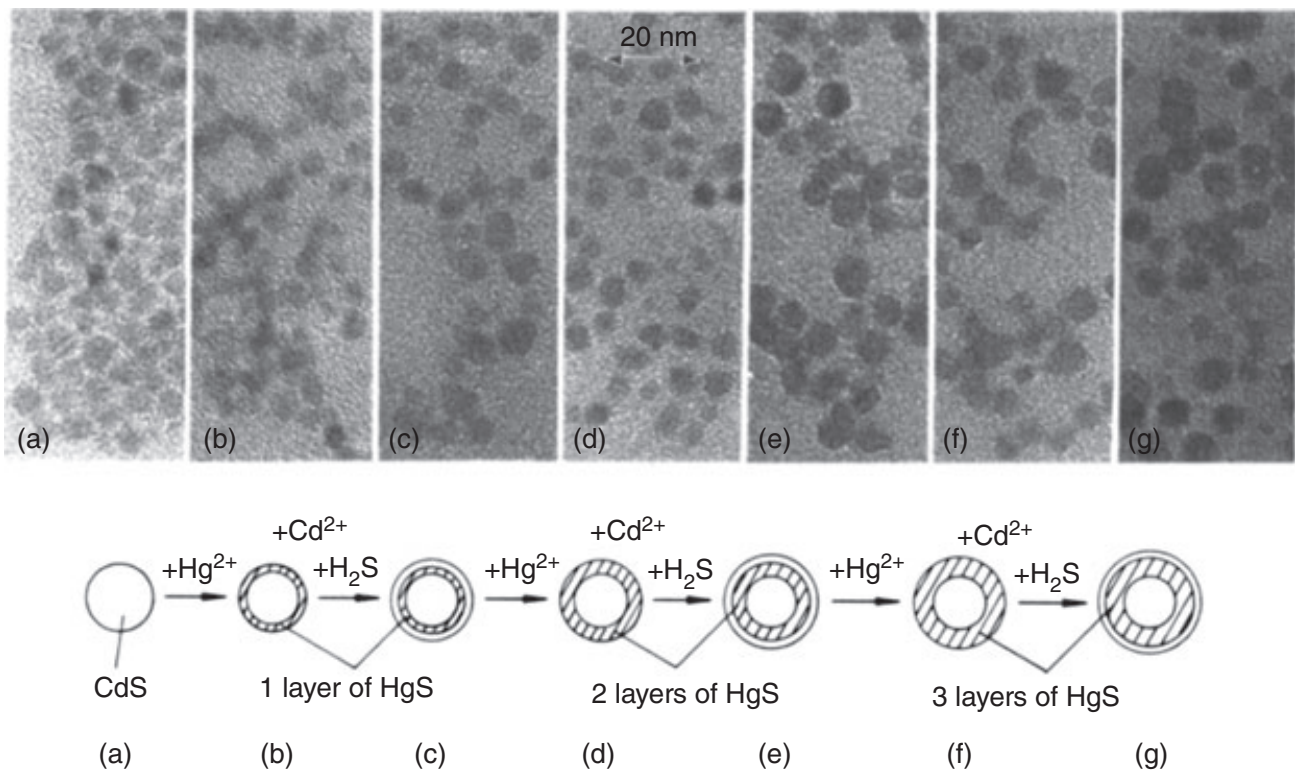

Figure 9.5 Scheme of the synthesis of $\mathrm{CdS} / \mathrm{HgS} / \mathrm{CdS}$ QDQWs and TEM images at various stages of the synthesis.

further increase in Cd-ion concentration occurred while the Hg-ion concentration suddenly began to rise. This indicated that only one ionic monolayer had been substituted. Subsequently, the Cd-ions released into solution could be reprecipitated onto the particles by the addition of $\mathrm{H}_{2} \mathrm{~S}$. The emerging colloidal particles consisted of a CdS core surrounded by a monolayer of HgS, and almost one monolayer of CdS as the outermost shell. From this point the preparation was seen to divide into two branches: (i) either increasing the HgS layer thickness; or (ii) increasing the thickness of the outermost CdS layer. The thickening of the HgS layer was achieved simply by repeating the substitution and reprecipitation steps described above. An increase in the CdS layer thickness was performed independently of the thickness of the formerly prepared HgS layer by the further addition of Cd-ions and precipitation of these onto the nanocrystals with $\mathrm{H}_{2} \mathrm{~S}$.

The spectral evolution in the course of the further preparation is depicted in Figure 9.6. The major finding was a strong shift of the absorption onset towards lower energies, with an increase in the thickness of the HgS well. This behavior could be explained with the small band gap of $\mathrm{HgS}(0.5 \mathrm{eV})$ with respect to $\mathrm{CdS}$ $(2.5 \mathrm{eV})$, and a localization of the charge carriers in the $\mathrm{HgS}$ wells. One remarkable finding was that the CdS capping of the particles also led to a significant shift of the absorption onset towards lower energies, even though CdS itself cannot absorb in this spectral region.

Theoretical calculations for these structures were performed by the same group in 1994 [15], applying the "particle in the box" model with the effective approximation resulting in calculated values for the first electronic transition and the cor- 


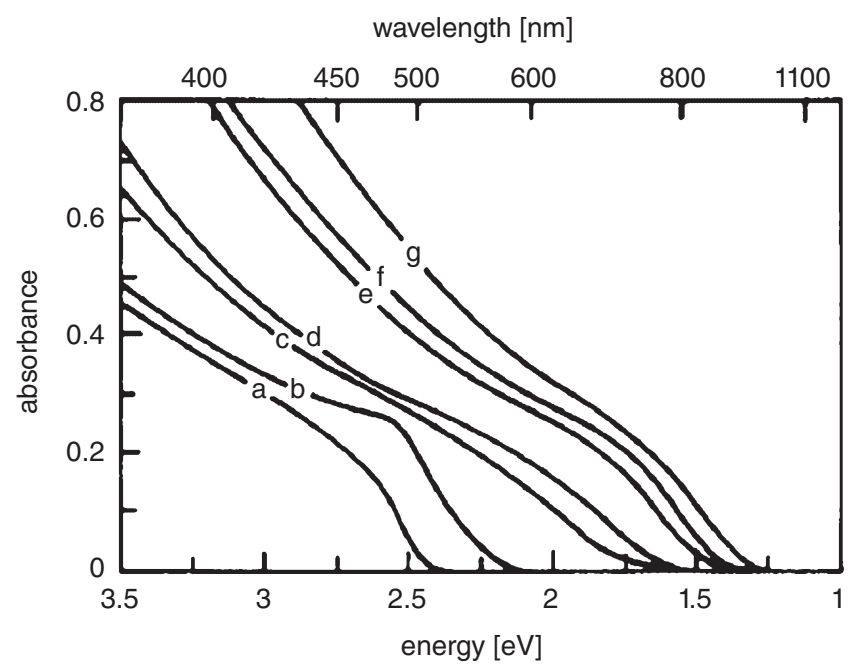

Figure 9.6 Absorption spectra of the colloidal solutions of (a) CdS, (b) a $+8 \times 10^{-5} \mathrm{M} \mathrm{Hg}^{2+}$, (c) b $+\mathrm{H}_{2} \mathrm{~S}$, (d) $\mathrm{c}+8 \times 10^{-5} \mathrm{M} \mathrm{Hg}^{2+}$, (e) $\mathrm{d}+\mathrm{H}_{2} \mathrm{~S},(\mathrm{f}) \mathrm{e}+8 \times 10^{-5} \mathrm{M} \mathrm{Hg}^{2+}$, and $(g) f+\mathrm{H}_{2} \mathrm{~S}$ (see Figure 9.5).

responding wave functions for the charge carriers. The presented results were in good agreement with the measured optical data. Further theoretical treatments were carried out by Bryant et al. after 1995 [16, 17].

Transient photobleaching experiments have been performed on these structures [18], with the most interesting result being that the photobleaching followed (spectrally) the newly evolving $1 \mathrm{~s}-1 \mathrm{~s}$ electronic transition of the composite particles. Some considerations concerning the charge carrier dynamics in the novel QDQWs were also outlined by these authors.

The subpicosecond photoexcitation of CdS/HgS/CdS QDQW nanoparticles at wavelengths shorter than their interband absorption $(390 \mathrm{~nm})$ leads to a photobleach spectrum at longer wavelengths $(440-740 \mathrm{~nm})$ [19]. The photobleach spectrum changes, and its maximum red-shifts with increased delay time. These results may be explained by the rapid quenching of the initially formed laserexcited excitons by two types of energy acceptor (traps); the first trap is proposed to be due to CdS molecules at the CdS/HgS interface, while the second trap is that present in the $\mathrm{CdS} / \mathrm{HgS} / \mathrm{CdS}$ well. The results of excitation at longer wavelengths, as well as the formation and decay of the bleach spectrum at different wavelengths, strongly support this description.

The homogeneous absorption and fluorescence spectra of the CdS/HgS QDQW system were also investigated using transient hole burning and fluorescence linenarrowing spectroscopy. Again, these photophysical measurements provided evidence for a charge-carrier localization within the $\mathrm{HgS}$ well [20].

High-resolution transmission microscopy (HRTEM) studies conducted with the CdS/HgS/CdS QDQW system have been performed to identify the details of the 
system's crystallography [21]. The HRTEM images of CdS nanocrystals showed triangular features, with the spacings and angles between the lattice planes showing alignment along the (110) axis of the zinc-blende crystal structure of CdS. The decrease in contrast when moving from the apex to the base implies a decrease in thickness which, in turn, suggests that the nanocrystal is a tetrahedron terminated in (111) surfaces. Corresponding HRTEM simulations agreed with this interpretation of the experimental image, although only a small fraction of the crystallites were aligned along the proper crystallographic axis to allow the shape to be discerned. The basic morphology was preserved in the next step of the synthesis, in which the surface cadmium ions of the CdS crystallites were exchanged with mercury. The final coating of the particles was carried out by adding excess cadmium ions to the solution and growing CdS on top of the HgS layer, via a slow $\mathrm{H}_{2} \mathrm{~S}$ injection. The close match of the $\mathrm{CdS}$ and $\mathrm{HgS}$ lattice parameters $\left(a_{\mathrm{HgS}}=5.852 \AA, a_{\mathrm{CdS}}=5.818 \AA\right)$ and the presence of faceted crystallites with only one exposed plane favored this growth mode.

The influence of the crystallography of the interface between the CdS and the $\mathrm{HgS}$ well on the optical properties of the CdS/HgS/CdS system was the subject of further characterization which employed optical detected magnetic resonance spectroscopy (ODMR) [22].

In the field of QDQW structures, a variety of new material combinations have also been used to prepare these structures. El-Sayed first reported on a ZnS/CdS QDQW system [23] in which the structures were characterized optically and the results compared with theoretical calculations. A QDQW system which consisted of CdS nanocrystals with an embedded monolayer of CdSe was presented by Battaglia et al. [24]. For this, the QDQW structures were prepared in high-boiling organic solvents using the SILAR technique (as introduced by Peng et al., cf. Ref. [6]), yielding almost monodisperse QDQW systems with high emission quantum efficiencies.

In 2001, the first results were reported by the present authors of an extended CdS/HgS QDQW with two embedded HgS wells [25], and also by the group of El-Sayed [26]. When further characterization of these structures were conducted using X-ray photoelectron spectroscopy [27] as a depth profiling technique, all results obtained were in good agreement with the predicted structure.

These structures are of interest as they allow studies to be made of the distancedependent interaction between two QWs within one QD. In a later report, the spectroscopic properties of these double-well QDs were also compared with theoretical calculations in the frame of the effective mass approximation [28]. Basically, the synthetic procedure for the double-well QD nanocrystals is the same as for the normal QDQW systems, with the outermost ion monolayer of the CdS nanocrystals being substituted with $\mathrm{HgS}$ by the addition of $\mathrm{Hg}\left(\mathrm{ClO}_{4}\right)_{2}$. The $\mathrm{Cd}^{2+}$-ions released into solution are then reprecipitated onto the particles by the addition of $\mathrm{H}_{2} \mathrm{~S}$. Different double-well QD systems can be obtained by a sequence of growing of CdS shells and substituting them by HgS. For ease of discussion, a nomenclature for the $\mathrm{CdS} / \mathrm{HgS} / \mathrm{CdS} / \mathrm{HgS} / \mathrm{CdS}$ samples is introduced according to Figure 9.7 as $\mathrm{CdS} / \mathrm{HgS}-\mathrm{ABCD}$, where each letter reflects the thickness of the correspond- 


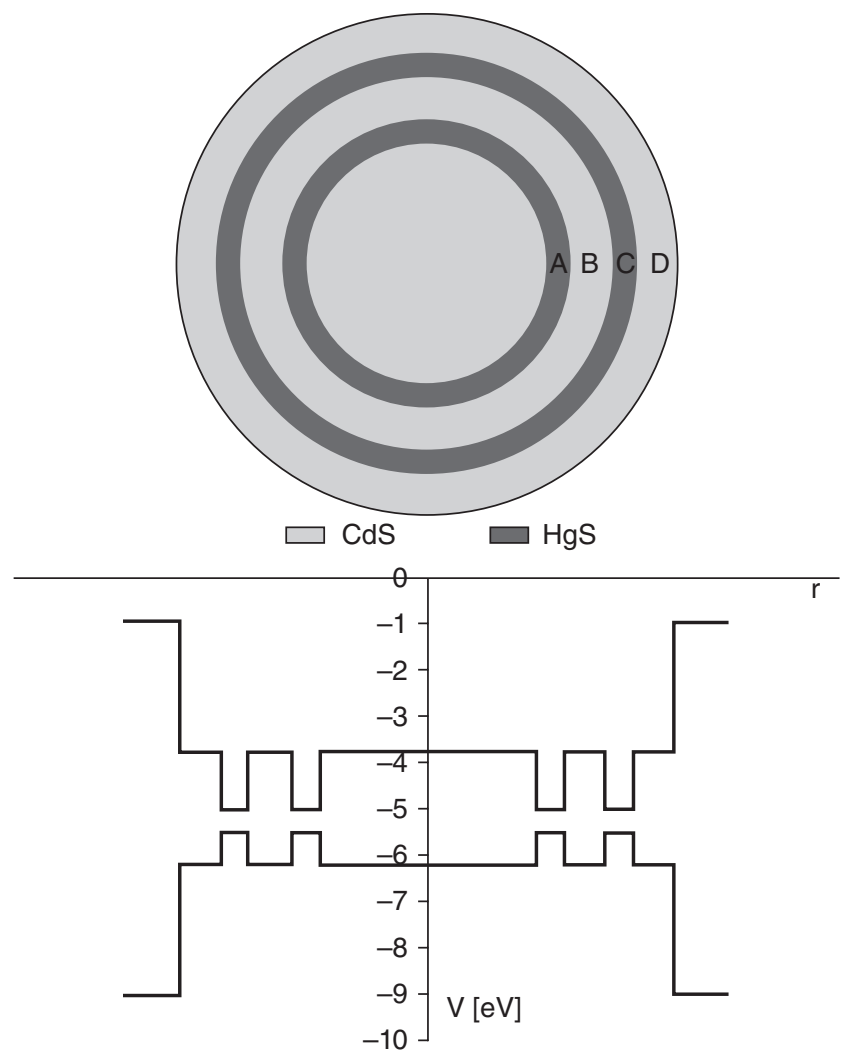

Figure 9.7 Idealized picture of the double-well QD structure with introduction of the CdS/HgS-ABCD nomenclature, and the corresponding radial potential for electron and hole [28].

ing layer in the monolayers. For example, CdS/HgS-1213 relates to a nanocrystal consisting of a CdS core, followed by one monolayer of $\mathrm{HgS}$, two monolayers of $\mathrm{CdS}$, one monolayer of $\mathrm{HgS}$, and again three monolayers of $\mathrm{CdS}$ as the outermost capping. CdS/HgS/CdS QDQWs are analogously named as CdS/HgS-AB. Of course, whilst this nomenclature refers to an idealized situation, in reality the particles will have variations in the thickness of all layers and are expected to exhibit inhomogeneities within the different layers.

Figure 9.8 displays the calculated radial probabilities of presence for the electron and the hole for the CdS/HgS- $1 \times 13$ series of nanocrystals, named according to the nomenclature described above, where $x$ is varied from $x=0$ to $x=7$. These nanocrystals all contain the same CdS core-two wells each consisting of one monolayer of $\mathrm{HgS}$, and three outer cladding layers of CdS. The only difference here is that the distance between the two $\mathrm{HgS}$ wells can be varied from zero to seven monolayers of CdS. As might be expected, the probability of presence has a maximum within the HgS wells for both the electron and the hole, by this giving rise to a 

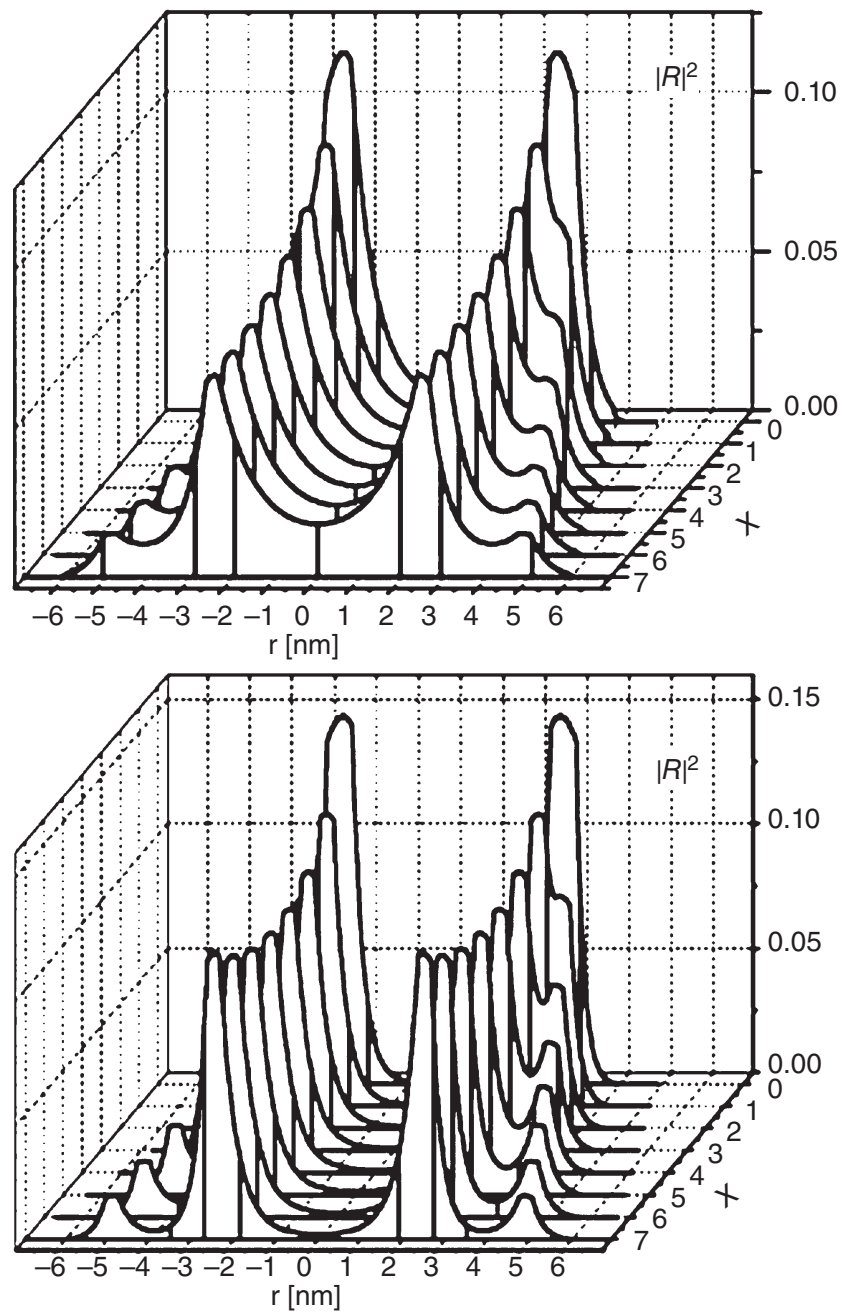

Figure 9.8 Radial probability of presence in the $\mathrm{CdS} / \mathrm{HgS}-1 x 13$

systems $(x=0-7)$ for the electron (top) and the hole (bottom), where $r$ is the radial distance from the particle center [28].

spatial overlap of the two wavefunctions within the $\mathrm{HgS}$ wells. In both materials the effective mass of the hole is larger than that of the electron, and therefore the localization is much stronger for the hole than for the electron in the same systems. Increasing the distance between the two $\mathrm{HgS}$ monolayers results in a more pronounced separation of the two maxima of the probability of presence; however, this separation becomes increasingly smeared out with a decrease in the distance of the two HgS layers. A similar behavior can be observed for the electron, although due to the smaller effective mass the maxima are not as well separated, 

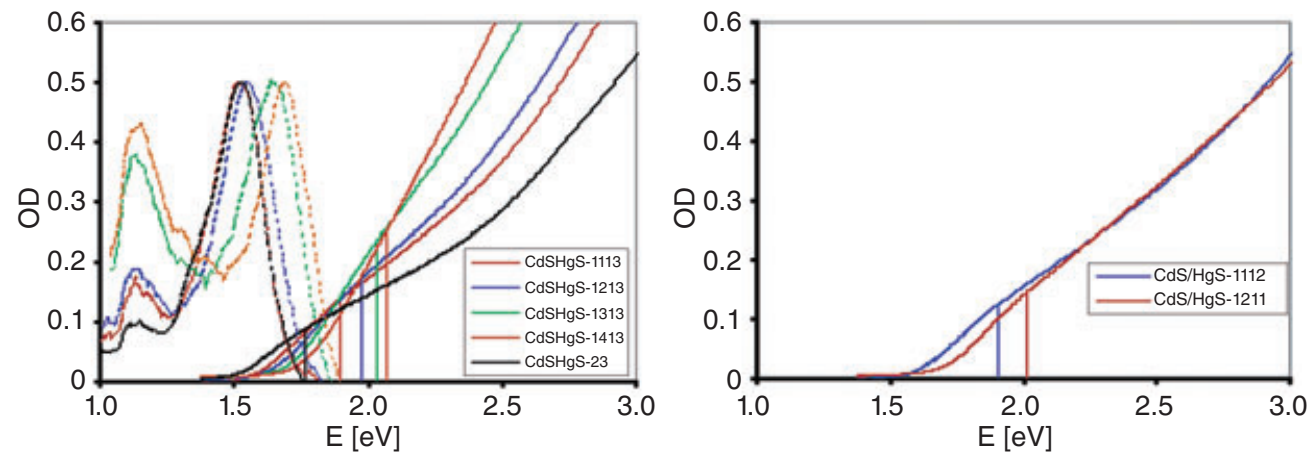

Figure 9.9 UV/Visible absorption and emission spectra (lines and dotted lines, respectively) of the CdS/HgS- $1 \times 13$ systems, together with the calculated first electronic transition (left) and the comparison of two systems with the same amount of $\mathrm{CdS}$ and $\mathrm{HgS}$ but different layer structures (right). Lines of the same color refer to the same sample in the left figure [28].

even for the system with the largest distance between the two HgS layers (i.e., CdS/HgS-1713).

Figure 9.9 (left) shows the UV-Vis absorption spectra of the CdS/HgS-1 113 series of nanocrystals, with $x$ ranging from 0 to 4 . Here, the vertical bars represent the calculated first electronic transition $\left(E_{\text {gap }}\right)$ for the corresponding idealized systems, while the point of maximum curvature is considered to represent the first electronic transition of the sample. For the samples CdS/HgS-23, CdS/HgS-1113, and $\mathrm{CdS} / \mathrm{HgS}-1213$, this point matches quite well with the calculated transition energies. In the case of the samples CdS/ HgS-1313 and CdS/HgS-1413 such a point is difficult to discern, but the absorption onset of those samples is still shifted towards higher energies, and is in good agreement with the calculated values (cf. the vertical bars). This may be interpreted by a decreasing interaction between the two HgS layers, but with an increasing distance between them. The high energy absorption (above $2.5 \mathrm{eV}$ ) depends mainly on the total amount of absorbing material. The absorption behavior between 1.8 and $2.2 \mathrm{eV}$ is not easily explained because this simple theoretical treatment is not suitable for explaining the oscillator strengths in different regions of the spectra. The dotted lines in Figure 9.9 (left) are the corresponding normalized emission spectra of the particles in these samples. Here, each sample shows an emission close to the band gap absorption onset; moreover, each sample shows a second emission at $1.15 \mathrm{eV}$ which is likely to be "trap emission." These traps are most probably related to stacking faults at the interfaces of the layers, as shown previously using ODMR measurements on the CdS/HgS/CdS systems [22]. In order to demonstrate that the absorption onsets of those systems are not only affected by the molar ratio of CdS to $\mathrm{HgS}$, but do indeed depend on the layer structure, a comparison of two structures with the same molar ratio but a different layer structure (namely, CdS/HgS-1112 and CdS/HgS-1211) is shown in Figure 9.9. The structure in which the HgS wells are 
separated by two monolayers of CdS, and with a capping layer of one monolayer, displays an absorption onset at a higher energy than that with a separation of one layer of CdS and two capping layers of CdS. In both cases, the agreement with the calculated transition energy is satisfactory.

The first report of a ternary core-shell/shell (CSS) structure was made by Reiss et al. in 2003, and included the details of a synthetic procedure that yielded CdSe/ ZnSe/ZnS CSS particles [29].

In 2004, Talapin synthesized similar particles [30], whereby CdSe nanocrystals capped with TOP ( $n$-trioctylphospine)/TOPO (n-trioctylphosphinoxide) were coated with a shell of either CdS or ZnSe. In both cases, the outermost shell was composed of ZnS, the main purpose of which was to avoid charge carrier migration towards the surface of the particles $(\mathrm{ZnS}$ is a good candidate due to its large bandgap of $3.7 \mathrm{eV}$ ). The large lattice mismatch between $\mathrm{CdSe}$ and $\mathrm{ZnS}$ was problematic, but this was overcome by the use of an intermediate ZnSe or CdS shell as a "lattice adapter."

Early reports on simple CdSe/ZnS core-shell particles showed that the quantum yield of the nanocrystals as a function of the ZnS layer thickness passed through a maximum at a ZnS layer thickness of approximately two monolayers [4]. The reason given for this behavior was the strain induced in the system by the lattice mismatch of ZnS and CdSe.

Figure 9.10 displays the basic structure of the CSS structures (panel a), together with the potential stepping of the valence and conduction band edges (panel b), and the band gaps of the materials as a function of the lattice spacing (panels $\mathrm{c}$ and d). It can be seen, that not only the band gaps but also the lattice spacing of $\mathrm{CdS}$ and $\mathrm{ZnSe}$ (the materials used as buffer layers) were between those of CdSe and $\mathrm{ZnS}$ and, therefore, both materials were well suited to act as "lattice adapters."

Figure 9.11 shows the development of the absorption and emission spectra during the coating procedure, together with the development of the quantum efficiency as a function of the shell thickness for different shell compositions. Remarkably, the shift to lower energies of the first absorption signal was stronger for the $\mathrm{ZnSe}$ coating than for the $\mathrm{ZnS}$ coating, which was interpreted as a stronger "leakage" of the wavefunction of the exciton into the ZnSe shell compared to the $\mathrm{ZnS}$ shell. According to these authors, another significant finding was that the quantum efficiencies of the CSS structures did not fall as strongly for an increased ZnS layer thickness as they did for "normal" CdSe/ZnS core-shell structures. This was attributed to a higher crystallinity as a consequence of stress release of the CSS structures compared to the CdSe/ZnS structure.

Another identified benefit of the CSS structures was their greatly enhanced photostability. For example, when single-particle luminescence images of CdSe/ ZnS core-shell structures and CdSe/ZnSe/ZnS CSS structures were recorded, after illumination with a laser beam under ambient conditions, almost all of the CSS structures were still luminescent after $10 \mathrm{~min}$, whereas most of the core-shell particles had already been extinguished. This situation was assigned to a higher stability against photo-oxidation of the CSS structures in comparison with the 
(a)

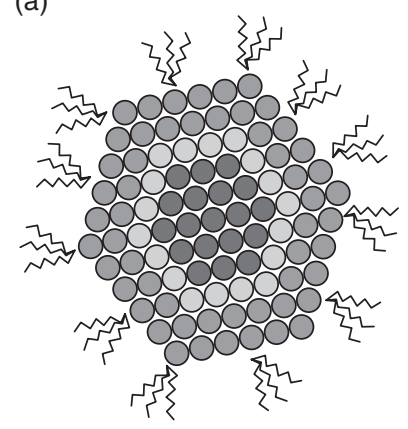

(b)
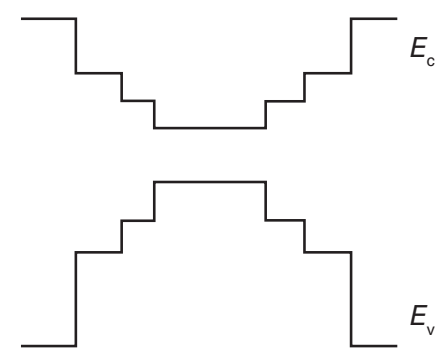

(c)

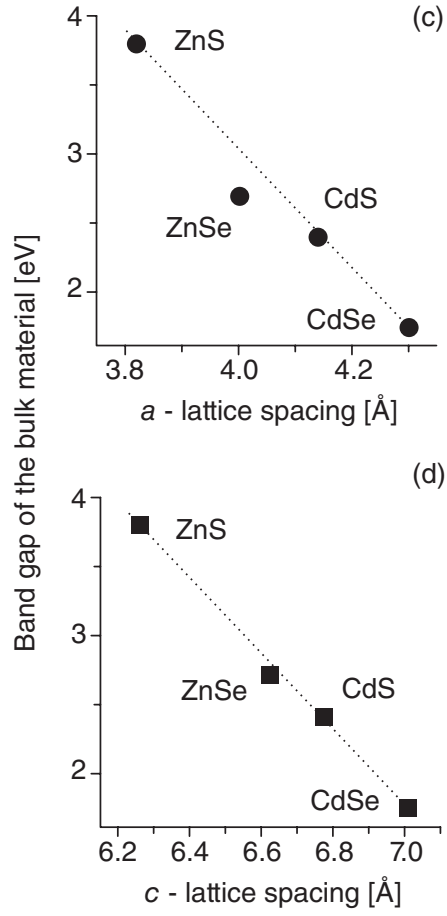

Figure 9.10 CSS nanocrystal. (a) Schematic outline; (b) The schematic energy level diagram; (c, d) Relationship between band gap energy and lattice parameter of bulk wurzite phase $\mathrm{CdSe}, \mathrm{ZnSe}, \mathrm{CdS}$, and ZnS [30].

core-shell particles. Recently, Jun et al. presented a simplified one-step procedure for the synthesis for CdSe/CdS/ZnS CSS nanocrystals [31].

In 2005, another example of CSS structures with a lattice-adapting layer was reported by Xie et al. [32]; in this case, the new structure was a CdSe/CdS/ZnS CSS structure (as presented above). However, the authors reported the possibility of including an alloyed layer of $\mathrm{Zn}_{0.5} \mathrm{Cd}_{0.5} \mathrm{~S}$ into the structure, resulting in $\mathrm{CdSe} /$ $\mathrm{CdS} / \mathrm{Zn}_{0.5} \mathrm{Cd}_{0.5} \mathrm{~S} / \mathrm{ZnS}$ multishell particles; they also reported on very high quantum efficiencies for the multishell particles. In addition, their results with regards to stability versus photo-oxidation showed the same trend as those noted by others [30].

Transmission electron microscopy (TEM) images presented elsewhere [32] (Figure 9.12) demonstrated the growth of the particles, and showed clearly that the particles had retained a very narrow size distribution throughout the coating procedure.

Recently, the group of Banin reported an example of a CSS structure with InAs, and thus a Group III-V core material [33]; these InAs cores were covered with an intermediate layer of CdSe and an outermost layer of ZnSe. As noted elsewhere for pure Group II-VI CSS particles, very high emission quantum efficiencies (up 


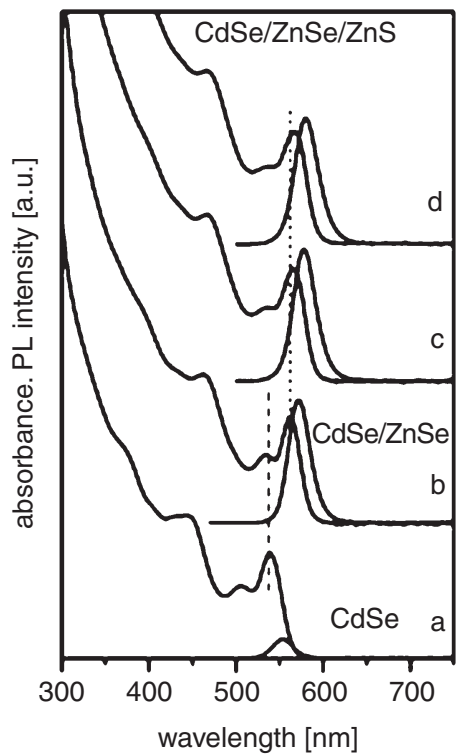

Figure 9.11 Left: Absorption and PL spectra of (a) CdSe cores, (b) CdSe/ZnSe core-shell nanocrystals (thickness of the ZnSe shell $\sim 2$ monolayers); (c, d) CdSe/ZnSe/ZnS nanocrystals with the thicknesses of the ZnS shell $\sim 2$ monolayers (c) and $\sim 4$ monolayers (d). Right: Room-temperature PL quantum

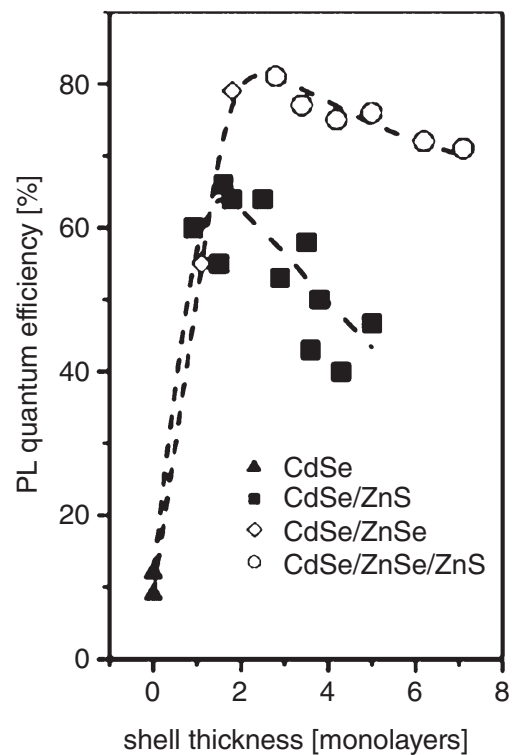

yields of CdSe, CdSe/ZnSe, and CdSe/ZnSe/ ZnS nanocrystals dissolved in chloroform. For comparison, the dependence of the PL quantum yield on the shell thickness for various samples of $\mathrm{CdSe} / \mathrm{ZnS}$ nanocrystals is displayed [30].

to $70 \%)$ were reported. However, due to the use of InAs as the core material, in this case the emission wavelength was tunable throughout the NIR region of the spectrum (800-1600 $\mathrm{nm}$ ). This report showed clearly that the properties of the CSS nanocrystals can neither be obtained with simple InAs/CdSe, nor with InAs/ZnSe core-shell nanocrystals. Figure 9.13 shows the evolution of the quantum yield as a function of the coating for InAs/CdSe core-shell nanocrystals, InAs/ZnSe nanocrystals, and the CSS system (where the first layer added is from CdSe and the outer shells are composed of $\mathrm{ZnSe}$ ). In the case of the InAs/CdSe system, a clear maximum of the quantum yield was observed for one monolayer of CdSe, whilst for the InAs/ZnSe particles the quantum efficiencies increased continuously but reached only about $15 \%$. In the CSS system, the quantum efficiencies increased continuously to about $50 \%$.

In summary, the CSS structures with a lattice-adapting intermediate layer have been proven to be superior systems with regards to their luminescence quantum yields and stability versus photo-oxidation.

Another interesting structure developed by Peng and coworkers was the CdSe/ $\mathrm{ZnS} / \mathrm{CdSe}$ CSS system [34]. As in this case the intermediate layer is composed of the high-band-gap material ZnS, these are not QDQW systems in the truest sense but, nevertheless, were of superior fundamental interest (cf. also Figure 9.12). This 

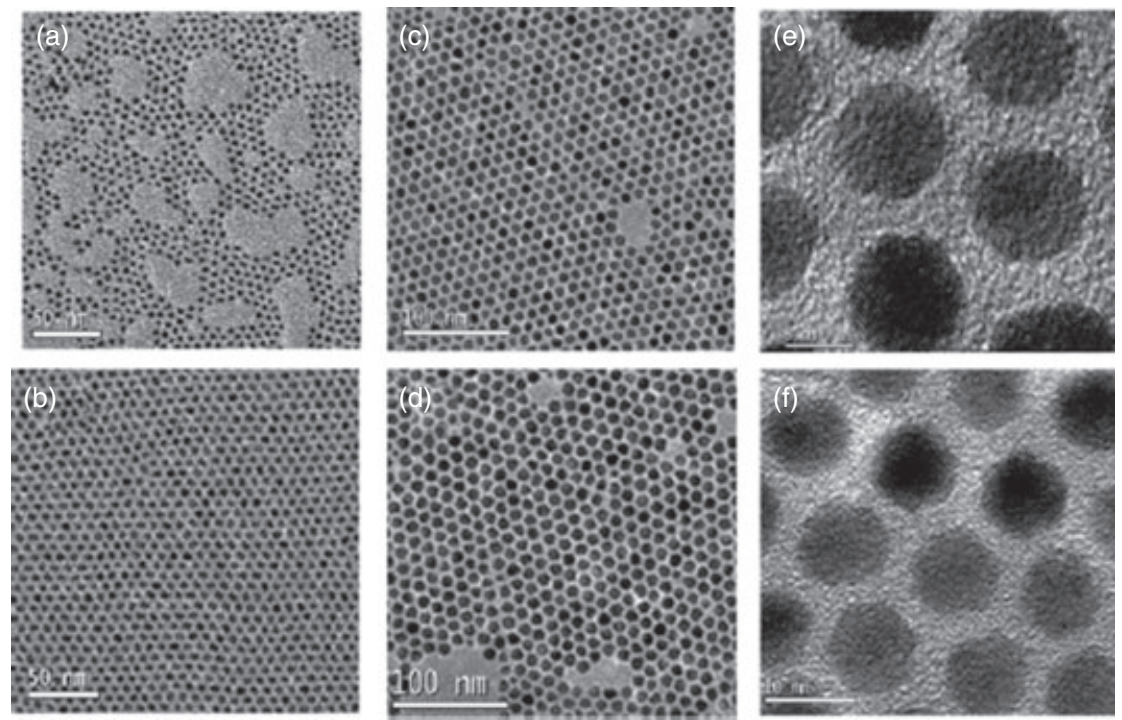

Figure 9.12 Transmission electron microscopy (TEM) images of the plain $\mathrm{CdSe}$ cores and core-shell nanocrystals obtained under typical reaction conditions. (a) TEM images of CdSe cores (before injection of $\mathrm{Cd}^{2+}$ solution); (b) (a) plus 2 monolayers of CdS; ( $d$ and e) (b) plus 3.5 monolayers of $\mathrm{Zn}_{0.5} \mathrm{Cd}_{0.5} \mathrm{~S}$; ( $\mathrm{d}$ and f) (c) plus 2 monolayers of $\mathrm{ZnS}$ [32].

report was the first to describe two distinct emission signals from one nanocrystal, where none of the signals was trap-related. The embedded ZnS layer seemed to be thick enough to completely decouple the outer shell of CdSe from the inner CdSe core of the particle. Figure 9.14 shows the evolution of the emission signal in this type of inversed QDQW. For particles with a ZnS layer of one monolayer thickness, an increase in the outermost CdSe QW led to a shift of the emission signal to lower energies, but not yet to the occurrence of a second emission signal. In contrast, at a ZnS layer thickness of three monolayers, no shift of the emission signal could be observed; rather, a second emission signal occurred at higher energies which was shifted to lower energies with increasing thickness of the outer CdSe well. This observation was explained as the phenomenon of "coupled and decoupled quantum systems in one semiconductor nanocrystal."

The shift towards lower energies for thin ZnS buffer layers can be interpreted as an interaction between the core and the outer shell; this is comparable to the interaction between the two QWs described above and referred to as a "double-well QD structure." The two distinct emission signals observed with thick intermediate ZnS layers are interpreted as a complete electronic decoupling of the outer CdSe $\mathrm{QW}$ and the CdSe core. These structures were subjected to further spectroscopic investigations [35] and also investigated as possible sources of white light emission [36]. However, as the presented measurements were ensemble in nature, it could not be excluded without doubt that in some, or all, cases the single nanocrystals 

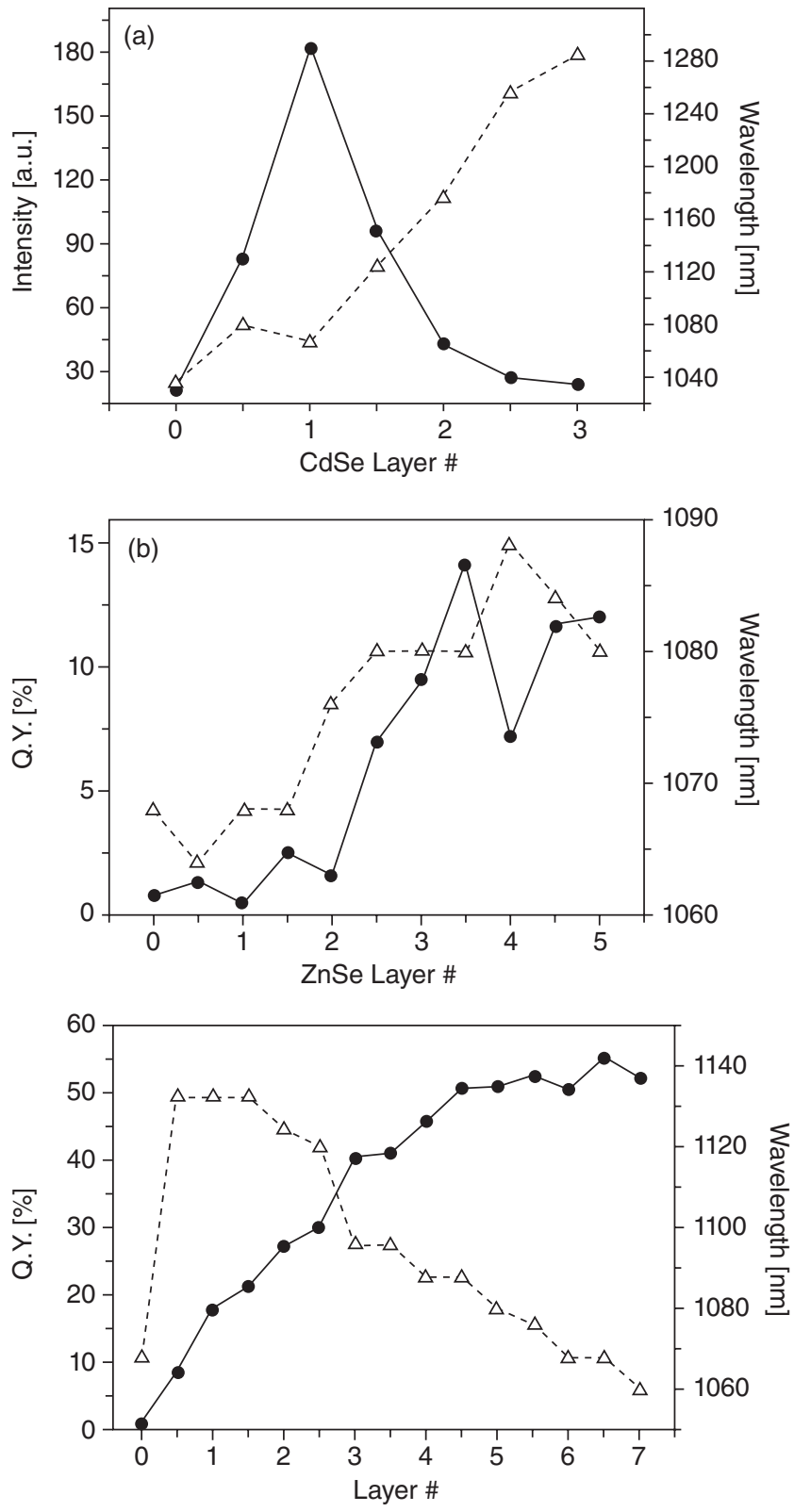

Figure 9.13 Quantum yields (black dots with full lines) and emission wavelengths (white triangles with dotted lines) as a function of the shell thickness in monolayers for $\operatorname{lnAs} / \mathrm{CdSe}$ core-shell nanocrystals (top), InAs/ZnSe core-shell nanocrystals (middle) and InAs/CdSe/ZnSe CSS nanocrystals (bottom, the first layer is CdSe, all other layers are ZnSe) [33]. 

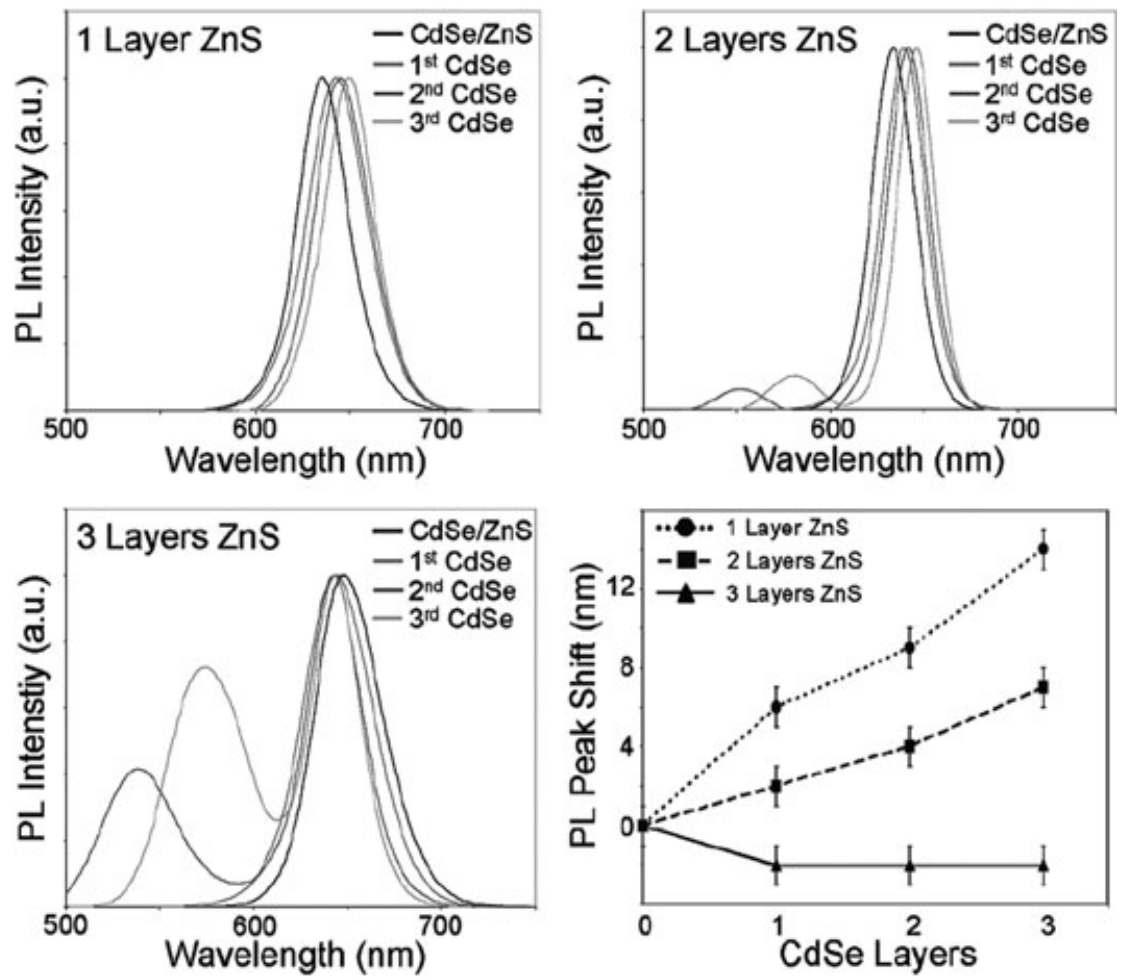

Figure 9.14 Emission spectra of $\mathrm{CdSe} / \mathrm{ZnS} / \mathrm{CdSe}$ nanocrystals with a ZnS barrier layer having the thickness of one (top left), two (top right) and three (bottom left) monolayers. All spectra are shown for the simple $\mathrm{CdSe} / \mathrm{ZnS}$ core-shell structure and for an outer shell of one, two, and three monolayers of CdSe. The variations of the positions of the emission maxima is summarized in the lower right figure [34].

had only an emitting core or an emitting shell, and not both. Only single-particle spectroscopy studies performed on these structures could ultimately resolve this question.

\subsection{4}

\section{Nonspherical Nanoheterostructures}

Following the development of synthetic routes towards an efficient shape control of homogeneous quantum rods [37], the development of nonspherical nanocrystals composed of more than one material was a logical step. The simplest approach here would be to overcoat a nanorod with an inorganic shell; this would result in a type-I band alignment and thus increased luminescence quantum yields (whilst retaining the polarized emission of the anisotropic quantum rod). An example would be CdSe quantum rods covered with $\mathrm{ZnS}$ [33], where the increase 
in fluorescence quantum yield could be explained in similar fashion to the case of spherical core-shell structures (reducing the probability of the presence of any charge carriers at the particle surface).

Other nonspherical heterostructures include compartment-like rods (e.g., CdTe/ CdSe and others [39]), and another class of nonspherical heterostructures which were started from QDs and the seeds used for the growth of a quantum rod. Following the pioneering studies of Talapin et al. [40], who synthesized elongated $\mathrm{CdSe} / \mathrm{CdS}$ core-shell structures, this approach has recently been further redeveloped by Talapin [41] and Manna [38], and has resulted in highly monodisperse, size- and aspect ratio-tunable CdSe seeded CdS nanorods (see Figure 9.15 for emission and absorption spectra and TEM images). One interesting aspect about this type of structure is that, especially for high-aspect-ratio rods, the absorption spectra of these heterostructures are dominated by the absorption of the CdS (since it is in 10- to 40-fold excess compared to $\mathrm{CdSe}$ ), while the emission arises only from the CdSe core (as the interband relaxation is much faster than the radiative recombination). Taking into account the fact that the photoluminescence excitation spectra also almost match the absorption spectra, it can be concluded that energy transfer from the rod towards the core is very efficient, and that the rod can be regarded as an antenna which absorbs light, while the emission of the light occurs always from the seed. This also means that light emitted by these seeded rods is much less reabsorbed than, for example, the case of pure CdSe rods. Consequently, such rods may attract interest for those applications where comparatively thick layers of nanocrystals are used, when the reabsorption should be limited as much as possible. The large-scale assembly of these seeded rods was also shown to be possible; this might be of special interest due to the capability of these structures to emit polarized light [38]. The seeded growth approach was recently applied to the development of other nonspherical heterostructures, such as ZnSe seeded CdS rods [42] (hence an anisotropic type-II structure with a charge carrier separation along the rod axes). These type-II nanorods show not only extended luminescence lifetimes but also a clearly spatially indirect transition with transition energies of around $2.1 \mathrm{eV}$ (thus much smaller than both bulk band gaps of $\mathrm{CdS}$ and $\mathrm{ZnSe}$, which were $2.5 \mathrm{eV}$ and $2.7 \mathrm{eV}$, respectively). A variety of seeded tetrapods was also synthesized using the seeded growth approach [43].

\section{3}

\section{Characterization of Nanoheterostructures}

Although, today, nanoheterostructures are widely accessible (e.g., CdSe/ZnS coreshell particles are available commercially from several sources), the characterization of these structures remains a major challenge for chemists and physicists. Whilst homogenous QDs can usually be sufficiently characterized with standard methods such as TEM, X-ray diffraction (XRD) and UV-Vis/NIR absorption and emission spectroscopy, further investigations are normally required for the (complete) characterization of heterogeneous quantum structures. Especially in the 


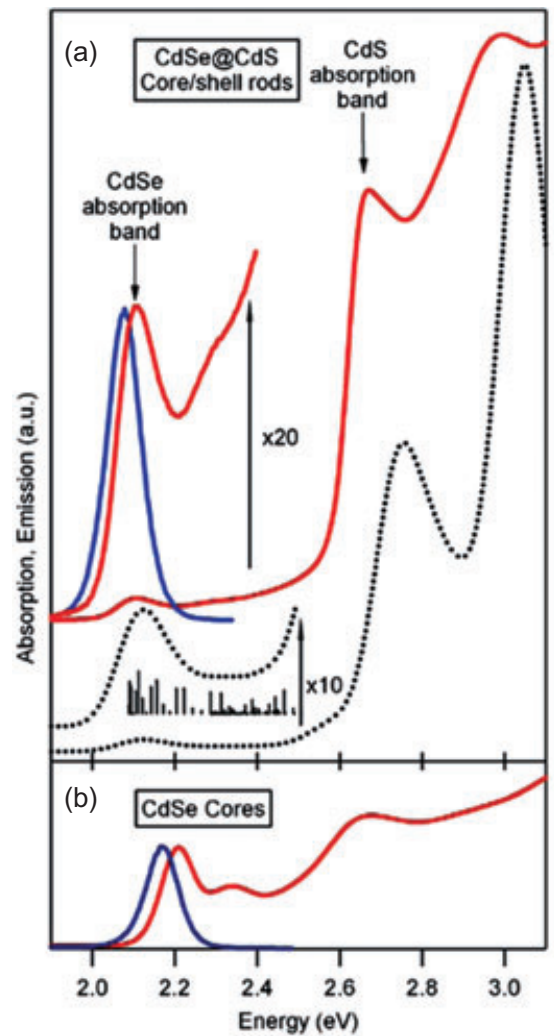

Figure 9.15 (a) Absorption (red) and emission (blue) spectra of CdSe-seeded CdS rods. The dotted line is a calculated spectrum; (b) Absorption and emission spectra of the CdSe seeds; (c-f) Transmission electron microscopy (TEM) images of CdSe-seeded $\mathrm{CdS}$ rods of different length and aspect ratios; (g) High-resolution TEM images of a CdSe-seeded CdS nanorod; (h) Highresolution TEM (HRTEM) images of pure CdS nanorod; (i, j) Corresponding "mean dilatation" images. This technique allows a mean dilatation mapping from high-resolution
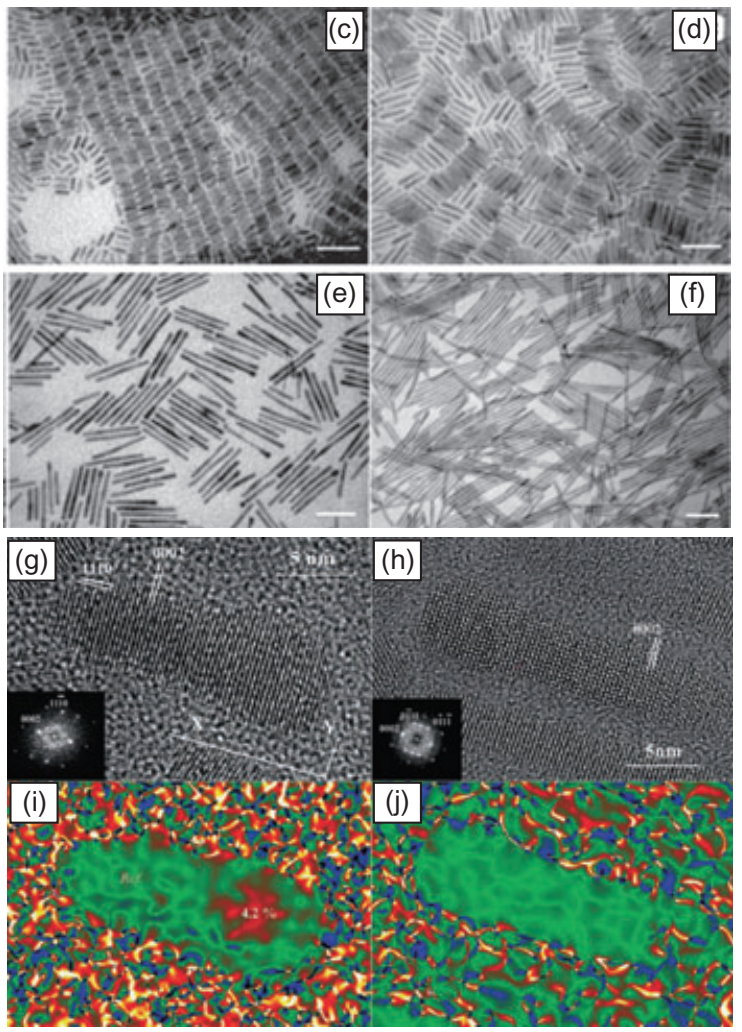

TEM images or, indeed, any type of lattice image. It uses a color scale for displaying variations in the periodicity of the HRTEM contrast. Areas of the same color are regions with the same periodicity. The mean dilatation image of the $\mathrm{CdSe} / \mathrm{CdS}$ rod (i) shows an area with lattice parameters altered by $4.2 \%$ with respect to the reference area, situated at the opposite tip of the rod. For comparison, the same analysis is performed on "CdS-only" rods, and no variation of the lattice parameters over the whole length of the nanorod can be observed (j) [38].

case of epitaxially grown shells, information about shell thicknesses and shell composition that can be obtained with crystallographic methods such as XRD, and to some extent also with HRTEM, are limited. A preliminary concept of the composition of a core-shell particle can be obtained by simply measuring the diameter of the original core particles and their diameter after shell growth. However, it must not be taken for granted that the original core particles do not grow by themselves under the shell growth conditions (e.g., via Ostwald ripening), and 
consequently carefully performed reference experiments and additional elemental analyses will be required to interpret the results. Even if diameter measurements, when combined with elemental analysis, might allow determination of the shell thickness, neither approach can solve the question of the degree of alloying in a core-shell structure. Bearing in mind that typical core-shell structures have shell thicknesses of around $0.5-2 \mathrm{~nm}$, the question of how much such a shell is alloyed (or not) seems almost impossible to answer. Further information might be obtained, however, by applying X-ray photoelectron spectroscopy. As the escape depth of the photoelectrons generated is typically in the range of just a few nanometers, thus is an extremely surface-sensitive technique. When using an energytunable X-ray source (e.g., a synchrotron), this escape depth can even be tuned to some degree and hence a depth profiling of a given core-shell structure is possible. Borchert et al. successfully applied the method not only to the characterization of simple CdSe/ZnS core-shell structures [44], but also to more sophisticated CdS/ HgS QDQW structures, as described above (cf. Ref. [27]).

\section{4}

\section{Core-Shell Infrared Nanoparticulate Materials}

The International Commission on Illumination (CIE) has recommended the division of longer-wavelength radiation into the following three bands: IR-A (700$1400 \mathrm{~nm})$; IR-B (1400-3000 nm); and IR-C (3000 $\mathrm{nm}$ to $1 \mathrm{~mm})$.

The following is a commonly used working scheme:

- Near-infrared (NIR, IR-A DIN): $0.75-1.4 \mu \mathrm{m}$ in wavelength, defined by the water absorption, and commonly used in fiber optic telecommunications because of low attenuation losses in the $\mathrm{SiO}_{2}$ glass (silica) medium. Applications utilizing this spectral region include image intensifiers, such as night-vision goggles.

- Short-wavelength infrared (SWIR, IR-B DIN): 1.4-3 $\mu \mathrm{m}$, water absorption increases significantly at $1450 \mathrm{~nm}$. The 1530 to $1560 \mathrm{~nm}$ range is the dominant spectral region employed for long-distance telecommunications.

- Mid-wavelength infrared (MWIR, IR-C DIN) also called intermediate infrared (IIR): $3-8 \mu \mathrm{m}$. In guided missile technology the 3-5 $\mu \mathrm{m}$ portion of this band is the atmospheric window in which the homing heads of passive IR "heat-seeking" missiles are designed to work, homing on to the IR signature of the target aircraft, typically the jet engine exhaust plume.

- Long-wavelength infrared (LWIR, IR-C DIN): $8-15 \mu \mathrm{m}$. This is the "thermal imaging" region, in which sensors can obtain a completely passive picture of the outside world based on thermal emissions only and requiring no external light or thermal source such as the sun, moon or infrared illuminator. Forwardlooking infrared (FLIR) systems use this area of the spectrum. It is sometimes also referred to as the "far-infrared".

- Far-infrared (FIR): 15-1000 $\mu \mathrm{m}$. 
Interest in NIR-active materials is primarily due to the fact that water is transparent across a number of wavelength ranges in this spectral region and, as a result, atmospheric transmission technologies and a number of biological applications can greatly benefit if these wavelengths are employed. The fact that there are no efficient and stable dyestuffs that can be used to address this spectral region had given extra impetus to the development of inorganic possibilities, and semiconductor nanocrystals have been demonstrated to adequately fulfill this role. Semiconductor nanocrystalline core materials exist that may be used directly to access this region, such as the Group IV-VI materials lead sulfide, lead selenide and lead telluride, as well as the Group III-V material InAs [45]. However, as the surface of nanoparticles is very sensitive to their immediate environment-a fact that usually results in a deterioration of the optical properties and, in particular, of the emission efficiency-it is as a rule beneficial to add a surface coating.

In order to achieve optical responses in the NIR, or to extend the spectral range of the nanocrystals further into the red, those materials of which the bulk bandgap is already reasonably narrow have been the main focus of investigation. A number of such systems have been reported, and have in general concentrated on the following as core materials: CdSe [46, 47], CdTe [48-54] or HgTe [55, 56] and the mixed ternary compounds $\mathrm{Cd}_{x} \mathrm{Hg}_{1-x} \mathrm{Te}$ [54] as Group II-VI materials, InP [57-59], InAs [33, 60-63] and GaAs [64-67] as Group III-V materials, and PbS [68], PbSe [69-76] and PbTe [77, 78] as Group IV-VI materials. Some mention of CSS systems [33, 46, 49, 60, 63] and different geometries such as tetrapods [47] and wires [64-67] have also been reported. It is worth noting at this point that there are a number of other core-shell material sets involving phosphors [79], transition metal oxides [80], noble metals [81] and their chalcogenides [82, 83], as well as various approaches to system design [84] and mixed nanoparticle-dyestuff compositions [85]. For example, although a CdSe/CdS core-shell system is not inherently an IR emitter, NIR emission can be achieved by coupling these nanocrystals to IR fluorescent dyestuffs. However, at this point attention will be focused on coreshell systems where the core is a QD composed of one of the Group II-VI, III-V, and IV-VI materials.

\section{5}

\section{Type I Core-Shell Infrared Structures}

Amongst the materials with which CdTe cores have been coated to produce type-I structures are ZnS [54] and InP/ZnS [49]. In addition, CdHgTe/ZnS core-shell nanocrystals which are highly luminescent, stable for months in butanol, have a higher resistance to photobleaching compared to cores, and which were synthesized using a hybrid approach (i.e., an aqueous-based synthesis, followed by transfer to organic solvents) have been reported by Tsay et al. These authors reported a high quantum yield (20-50\%) in the NIR wavelength region $(>700 \mathrm{~nm})$, a region which is attractive for various biological applications because of the reduced autofluorescence background, improved penetration into scattering tissues, and enhanced photostability. In order to demonstrate their biological utility, the 
CdHgTe/ZnS core-shells were coated with phospholipid micelles. This coating is known to suppress the toxicity of the nanocrystals (NCs) and also to render them water-soluble, so as to allow bioactivity and keep the quantum yields high [86]. Studies on IR-active core-shell $\mathrm{HgTe} / \mathrm{Hg}_{x} \mathrm{Cd}_{1-x} \mathrm{Te}(\mathrm{S})$ particles, the objectives of which were to elucidate the nature of the recombination emission being associated with a $\mathrm{Cd}-\mathrm{Hg}$ mixed site and structural information on the NC core-shell interface, have also been reported [55].

Indium phosphide nanoparticles have also been a popular and productive choice of material for study, as this Group III-V material has a bulk bandgap of $1.35 \mathrm{eV}$ $(\sim 920 \mathrm{~nm})$ [57] and a reduced toxicity in comparison to the Groups II-VI and IV-VI materials. However, to date InP (in fact, all of the Group III-V materials) have remained synthetically very challenging since, even when proving possible to synthesize, they usually possessed a low quantum yield that could be increased by coating the material with a wider band gap material. The coating materials of choice have been the Group II-VI semiconductors ZnS and ZnSe, and also with CdSe to result in a type-II structure [57-59], due to their favorable band offsets and lattice mismatches (see Figure 9.16). In studies conducted by Shu and colleagues, a simple method was reported for the synthesis of highly luminescent InP NCs with quantum yields of up to $30 \%$, and InP/ZnS NCs with quantum yields of up to $60 \%$. This method allowed the preparation of both InP and InP/ ZnS NCs within $20 \mathrm{~min}$, and no size-selection process was required. The NCs were

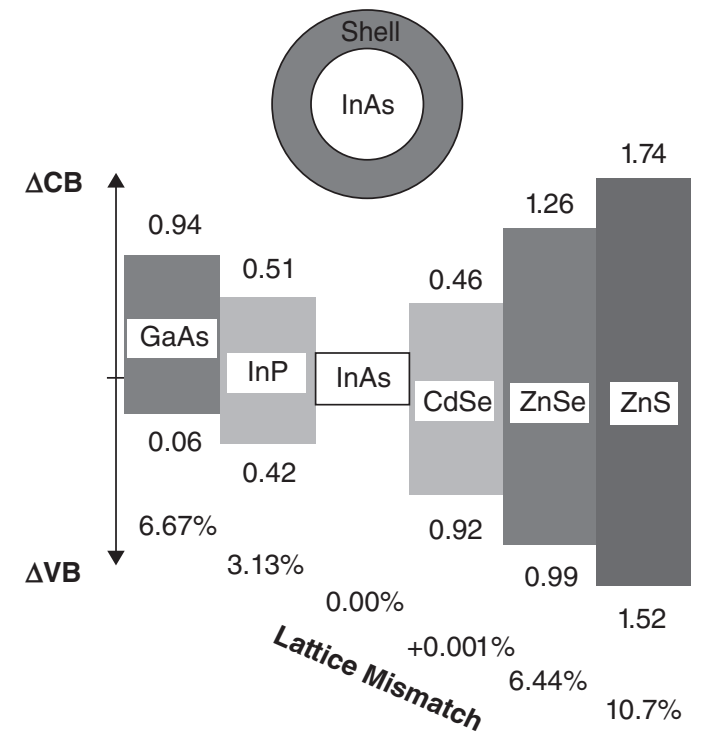

Figure 9.16 Summary of the band offsets (in eV) and lattice mismatch (in \%) between the core InAs and a number of III-V semiconductor shells (left side), and II-VI semiconductor shells (right side). $C B=$ conduction band; $\mathrm{VB}=$ valence band. Reproduced from Ref. [59]. 
obtained by a reacting $\mathrm{InCl}_{3}$ complex and tris(trimethylsilyl)phosphine in octadecene in the presence of zinc undecylenate. The photoluminesence spectra of differently sized InP/ZnS core-shell nanocrystals were presented that displayed peak maxima from 480 to $735 \mathrm{~nm}$. In the studies of Langhof and coworkers, a similar synthetic strategy was employed, using both $\mathrm{InCl}_{3}$ and tris(trimethylsilyl) phosphine, but the solvent mixture was a more coordinating solvent mixture that consisted of TOP/TOPO. The ZnS shell was achieved by transferring the NCs to pyridine and overcoating with the $\mathrm{ZnS}$ in this solution at $100^{\circ} \mathrm{C}$. The $\mathrm{ZnS}$ shell was synthesized by the reaction of diethylzinc and hexylmethyldisilathiane in tributylphosphine.

In this study, linearly polarized photoluminesence measurements showed that the InP/ZnS NCs studied had an elongated shape and an ellipsoidal eccentricity of about 0.6. Optical pumping photoluminesence decay curves revealed the spin relaxation time to be substantially shorter than the radiative lifetime of an exciton in InP/ZnS NCs, while the magnetic field-induced circularly polarized photoluminesence measurements supplied information about the g-factor of the exciton, electron, and hole. In studies performed by Banin et al., core-shell semiconductor nanocrystals with InAs cores were synthesized, and onto these Group III-V semiconductor shells (InP and GaAs), and Group II-VI semiconductor shells (CdSe, $\mathrm{ZnSe}$, and $\mathrm{ZnS}$ ) had been overgrown on cores of various radii, employing a two-step procedure. During initial attempts to carry out growth of the Group III-V semiconductor shell materials (InP, GaAs) it was found that these shells could be grown only at higher temperatures $\left(>240^{\circ} \mathrm{C}\right)$. In contrast to the Group III-V semiconductor shells, however, growth of the Group II-VI semiconductor shells (CdSe, ZnSe, $\mathrm{ZnS}$ ) was observed at the comparatively lower temperature of approximately $150^{\circ} \mathrm{C}$.

Yet, it was found that when growing Group II-VI semiconductor shells, a higher temperature was needed to increase the fluorescence quantum yield. For shell growth at $260^{\circ} \mathrm{C}$, the maximum fluorescence quantum yield of the products was about fourfold larger than that obtained at $160^{\circ} \mathrm{C}$. In addition, the comparison was noted between core-shell nanocrystals with InAs and CdSe cores. InAs/CdSe and $\mathrm{CdSe} / \mathrm{CdS}$, the core-shells were analogous in their electronic structure and hence, in both of these cases, because of the relatively low conduction band offsets, the electron wave function extended into the shell and to the nanocrystal surface; this in turn caused the spectrum to red-shift upon shell growth. Additionally, InAs/ $\mathrm{ZnSe}$ and InAs/ZnS core-shell materials were analogous to CdSe/ZnS. In this study, the band gap was barely changed upon shell growth, on account of the large band offsets between the core and the shell semiconductors. An overview of the absorbance and emission trends for the coating materials is shown in Figure 9.17.

\section{6}

\section{Type II Core-Shell Infrared Structures}

Included amongst the shells employed in the case of CdSe are CdTe [47] and $\mathrm{CdTe} / \mathrm{ZnTe}[46]$. In most cases, coating with CdTe is reported to yield a type-II 


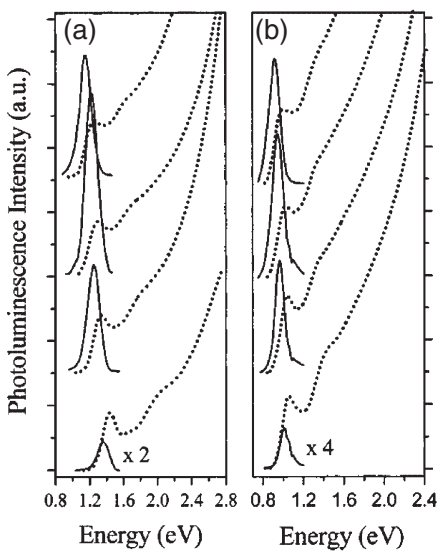

Figure 9.17 Evolution of absorption (dotted lines), and photoluminescence (solid lines) for growth of core-shells. The PL spectra are given on a relative scale for comparison of the enhancement of QY with shell growth. (a) InAs/CdSe with an initial core radius of $1.2 \mathrm{~nm}$. The shell thickness (in number of monolayers, $\mathrm{ML}$ ) and QY for the traces from bottom to top were respectively: $0,1.2 \%$; 0.6 , 13\%; 1.2, 21\%; 1.8, 18\%; (b) InAs/CdSe with initial core radius of $2.5 \mathrm{~nm}$. The shell thickness (in number of ML) and QY for the traces from bottom to top were respectively: 0, 0.9\%; 0.7, 11\%; 1.2, 17\%; 1.6, 14\%; (c) InAs/ZnSe with an initial core radius of $1.2 \mathrm{~nm}$. The shell thickness (in number of $M L$ )
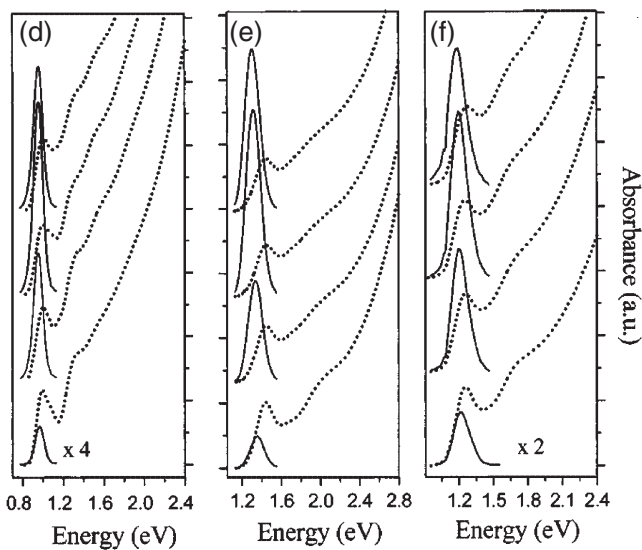

and QY for the traces from bottom to top were respectively: $0,1.2 \% ; 0.6,9 \% ; 1.5,18 \%$; 2.5, 14\%; (d) InAs/ZnSe with an initial core radius of $2.8 \mathrm{~nm}$. The shell thickness (in number of $\mathrm{ML}$ ) and QY for the traces from bottom to top were respectively: $0,0.9 \% ; 0.7$, 13\%; 1.3, 20\%; 2.2, 15\%; (e) InAs/ZnS with an initial core radius of $1.2 \mathrm{~nm}$. The shell thickness (in number of $\mathrm{ML}$ ) and QY for the traces from bottom to top were respectively: 0, 1.2\%; 0.7, 4\%; 1.3, 8\%; 1.8, 7\%; (f) InAs/ $\mathrm{ZnS}$ with an initial core radius of $1.7 \mathrm{~nm}$. The shell thickness (in number of $\mathrm{ML}$ ) and $\mathrm{QY}$ for the traces from bottom to top were respectively: $0,1.1 \% ; 0.6,5 \% ; 1.3,7.1 \% ; 2.2$, $6.3 \%$. Reproduced from Ref. [59].

system, but in a number of studies $[87,88]$ this was reported as type-I. This fact may be explained by a relative shift in the bands as the thickness of the coating layer was increased. Interestingly, in the CdSe/CdTe/ZnTe system, when the CdTe was first used as a coating material, an emission signal at $1027 \mathrm{~nm}$ was observed; yet, when it was employed as a sandwich layer the emission was observed to shift to between 1415 and $1470 \mathrm{~nm}$, depending on the thickness of the ZnTe layer $(1.3 \mathrm{~nm}$ to $1.8 \mathrm{~nm}$ ) (see Figure 9.18). In a second approach, the CdSe core size was altered from 3.4 to $5.7 \mathrm{~nm}$, while the shell thicknesses of both CdTe and ZnTe remained unchanged (within experimental uncertainty); as a result, the emission red-shifted from 1415 to $1518 \mathrm{~nm}$. These results correlated well with the band offsets of the ZnTe valence and the CdSe conduction band edges, and the resultant decrease of the $\mathrm{CdSe} \rightarrow \mathrm{ZnTe}$ transition. It was also noted that, in the case of the CdSe/CdTe/ZnTe structure (3.4/1.8/1.3 nm), a lifetime of up to $150 \mathrm{~ns}$ was observed for the CdSe $\rightarrow \mathrm{ZnTe} 1415 \mathrm{~nm}$ emission. This result further indicated a very long radiative lifetime of $\approx 10 \mathrm{~ms}$, due to the spatial separation of electron and hole by the CdTe intermediate layer. 


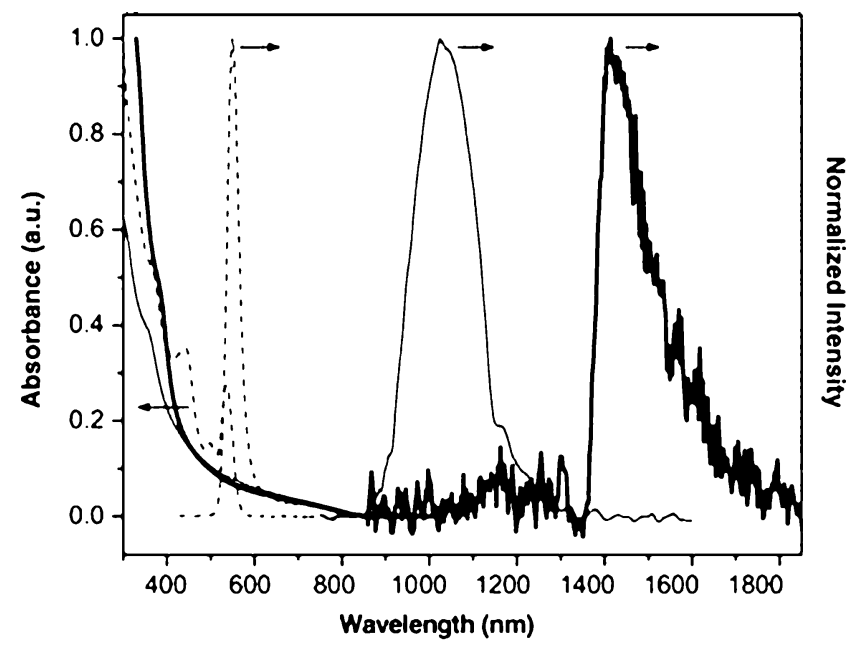

Figure 9.18 Normalized absorption and emission spectra of CdSe core $(3.4 \mathrm{~nm}$, dashed gray line), CdSe/CdTe $(3.4 / 1.8 \mathrm{~nm})$ core-shell (solid gray line), and $\mathrm{CdSe} / \mathrm{CdTe} / \mathrm{ZnTe}$ $(3.4 / 1.8 / 1.3 \mathrm{~nm})$ core-shell-shell (solid black line) QDs in toluene. Reproduced from Ref. [46].

Included amongst the materials that CdTe cores have been coated with to produce type-II structures are CdS [48] and CdSe [50-53]. For CdS-coated nanoparticles, the size distribution was observed to remain quite narrow upon coating with shell thicknesses of one to five monolayers. However, with increasing shell thickness the exciton lifetimes were seen to become longer (the average lifetime of the CdTe cores was $11.9 \mathrm{~ns}$ ). For CdTe/CdS structures with two- and four-monolayerthick shells, the time was increased to 23.2 and $26.8 \mathrm{~ns}$ respectively, while the slightly nonexponential decay curve observed for the bare CdTe core shifted to a monoexponential exciton decay, and was also accompanied by an increase in the photoluminescence quantum yields. In studies conducted by Kairdolf et al. on $\mathrm{CdTe} / \mathrm{CdSe}$ core-shell nanocrystals, a one-pot procedure for the synthesis of CdTe core QDs, prepared using multidentate polymer ligands in situ, resulted in a series of monodisperse CdTe QDs that showed a bright fluorescence from 515 to $655 \mathrm{~nm}$ (green to red). Fluorescence emission spectra showing the transition from CdTe cores to $\mathrm{CdTe} / \mathrm{CdSe}$ core-shell QDs, the emission of which was red-shifted from the original QD core emission of $650 \mathrm{~nm}$ to the NIR at $810 \mathrm{~nm}$ were presented. The absorbance spectra showed the red-shifting and eventual loss of the first exciton peak as the CdSe shell was grown on the CdTe core, typical of type-II QDs. Elsewhere [51], an aqueous route to $\mathrm{CdTe} / \mathrm{CdSe}$ materials with moderate fluorescence quantum yields (10-20\%) has been presented in which the uncoated CdTe displays an emission maximum at just below $650 \mathrm{~nm}$, while the CdSe-coated cores have an emission peak maximum at approximately $760 \mathrm{~nm}$. The lifetimes were also found to show more monoexponential decays and longer lifetimes as the CdSe shell was increased in thickness. These authors demonstrated the ability of the core-shell 
materials to selectively sense $\mathrm{Cu}(\mathrm{II})$ ions in the presence of other physiologically relevant cations in solution, by the quenching of the NIR fluorescence. Seo et al. observed that the presence of the alkyl metal precursors ethyl zinc or ethyl aluminum during the coating procedure led to rectangular shapes of CdTe/CdSe type-II dots, but spherically shaped materials could not be obtained. It was suggested that this synthesis proceeded by a mechanism in which the selenide anion, which is activated by ethyl zinc or ethyl aluminum, subsequently proceeded to react with excess cadmium cations. The absorption and emission spectra of two differently sized CdTe cores and their nonspherical core-shells, red-shifted from $692 \mathrm{~nm}$ and $751 \mathrm{~nm}$ to $760 \mathrm{~nm}$ and $802 \mathrm{~nm}$, respectively, which was to be expected for type-II dots. In subsequent studies based on femtosecond upconversion techniques, Chou and coworkers reported the first observation of early relaxation dynamics on CdTe/CdSe type-II QDs interband emission. CdTe/CdSe QDs, in which the core (core-shell) sizes, as measured with TEM size histograms were calculated as 5.3 (6.3), 6.1 (7.1), and 6.9 (7.8) nm, were used in the study. Upon an increase in diameter of the CdTe cores from 5.3 to $6.9 \mathrm{~nm}$, the CdTe emission peak wavelength-shifted from $690 \mathrm{~nm}$ to $737 \mathrm{~nm}$, while the emission of CdTe/ CdSe, coated with a similar thickness $(1.0 \mathrm{~nm})$ of CdSe, showed a similar systematic bathochromic shift from $1025 \mathrm{~nm}$ (core $5.3 \mathrm{~nm} /$ shell $1.0 \mathrm{~nm}$ ) to $1061 \mathrm{~nm}$ (core $6.9 \mathrm{~nm} / \mathrm{shell} 0.9 \mathrm{~nm}$ ). The relaxation results presented indicated that the electronseparation rate decreased as the size of the cores increased. In the absence of any observation of coherent optical phonon modes for both CdTe core (in CdTe/ $\mathrm{CdSe}$ ) emission and CdTe/CdSe interband emission, it is possible that the finite rate of charge separation might be due to the small electron-phonon coupling, causing a weak coupling between the initial and charge-separated states. The study results indicated that, in particular, the degree of control of the rate of electron transfer might prove useful in applications where a rapid carrier separation followed by charge transfer into a matrix or electrode was important, such as in photovoltaic devices.

An example of InAs $\mathrm{s}_{x} \mathrm{P}_{1-x} / \mathrm{InP} / \mathrm{ZnSe}$ III-V alloyed core-shell QDs, the bandgap of which has been engineered for applications in the NIR, has been reported by the group of Bawendi. The synthetic procedure for the alloyed core used the indium acetate, tris(trimethylsilyl)phosphine, and tris(trimethylsilyl)arsine as indium, phosphorus, and arsenic precursors, respectively. The resultant alloyed dot cores were overcoated with a shell of InP so as to increase their size and quantum yield. This shell was grown by injecting a mixture of indium acetate and tris(trimethylsilyl)phosphine to a solution of alloyed dots at $140^{\circ} \mathrm{C}$, which was low enough to avoid nucleation. The temperature was then raised to $180^{\circ} \mathrm{C}$ so as to initiate shell growth. Two successive injections of In and P precursors, both at $140^{\circ} \mathrm{C}$, to a solution of $\operatorname{InAs}_{0.82} \mathrm{P}_{0.18}(738 \mathrm{~nm}$ fluorescence peak) QDs resulted in core-shell particles with an emission at $765 \mathrm{~nm}$ after the first injection (full-width, half-maximum; FWHM $103 \mathrm{~nm}$ ) and $801 \mathrm{~nm}$ after the second injection (FWHM $119 \mathrm{~nm}$ ) (Figure 9.19), and a tripling of the QY.

These NIR-emitting CSS InAs $\mathrm{P}_{1-x} / \mathrm{InP} / \mathrm{ZnSe}$ were then subsequently successfully used in a sentinel lymph node (SLN) mapping experiment. When injected 


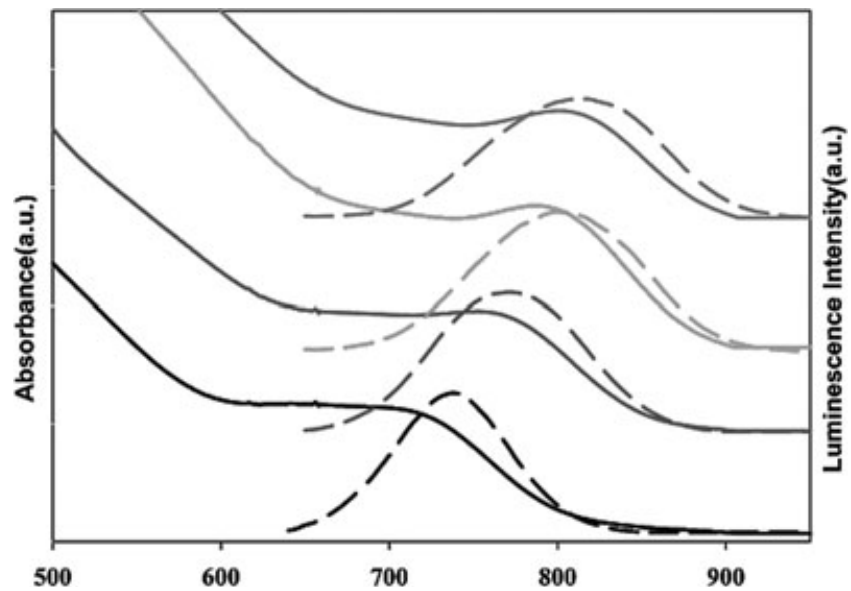

Figure 9.19 Absorbance (solid line) and corresponding photoluminescence (dashed line) spectra of core $\operatorname{InAs} s_{0.82} P_{0.18}$ QDs (emission $738 \mathrm{~nm}$, FWHM $86 \mathrm{~nm}$ ), after a first shell of InP (emission $765 \mathrm{~nm}$, FWHM $103 \mathrm{~nm}$ ), after a second shell of InP (emission $801 \mathrm{~nm}$, FWHM $119 \mathrm{~nm}$ ), and after the final ZnSe shell (emission $815 \mathrm{~nm}$, FWHM $120 \mathrm{~nm}$ ), from bottom to top, respectively. Reproduced from Ref. [63].

intradermally into the paw of a mouse, the small amount and low concentration of NIR QDs injected could not be seen on the color video images. However, the fluorescence image revealed the fine detail of lymphatic flow from the injection site to the SLN. As the background autofluorescence from the tissue was low in this spectral region, this permitted a high signal-to-background ratio.

The Group IV-VI lead chalcogenide semiconductor nanocrystals $\mathrm{PbS}, \mathrm{PbSe}$, and PbTe all form type-II core-shell structures with one another, and also have the advantage of possessing narrow bulk bandgaps $(0.41,0.278$, and $0.31 \mathrm{eV}$ at room temperature and $0.29,0.17$, and 0.19 at $4 \mathrm{~K}$, respectively) [45]. The band gap of $\mathrm{PbS}$ may be tuned to $800 \mathrm{~nm}$ in the absorbance [89], resulting in a set of materials that can be engineered to address the spectral range between $800 \mathrm{~nm}$ (quantumconfined $\mathrm{PbS}$ ) to beyond $7000 \mathrm{~nm}$ (bulk PbTe). The applications (as opposed to academic) interest in coating in the case of lead chalcogenides was primarily one of protecting the core, as the complete NIR region is accessible to these materials without any requirement for coating. Elsewhere, $\mathrm{PbS}$ has been coated with $\mathrm{PbSe}$ [68], and $\mathrm{PbSe}$ with $\mathrm{PbS}$ [70-76].

In the latter cases, $\mathrm{PbS}$ would be expected to be more robust to atmosphericinduced oxidation than would the PbSe core. The SILAR (Successive Ion Layer Addition and Reaction) technique has been applied to add up to five monolayers, with a consequent shift in absorbance of $85 \mathrm{~nm}$ for the coating of a $6 \mathrm{~nm}$ core. In the group of Lifshitz, the synthesis of both core-shell and core-alloyed shell has been undertaken, and the materials evaluated as optical components in Q-switching lasers. In this way, the synthesis of the core materials in the size range 

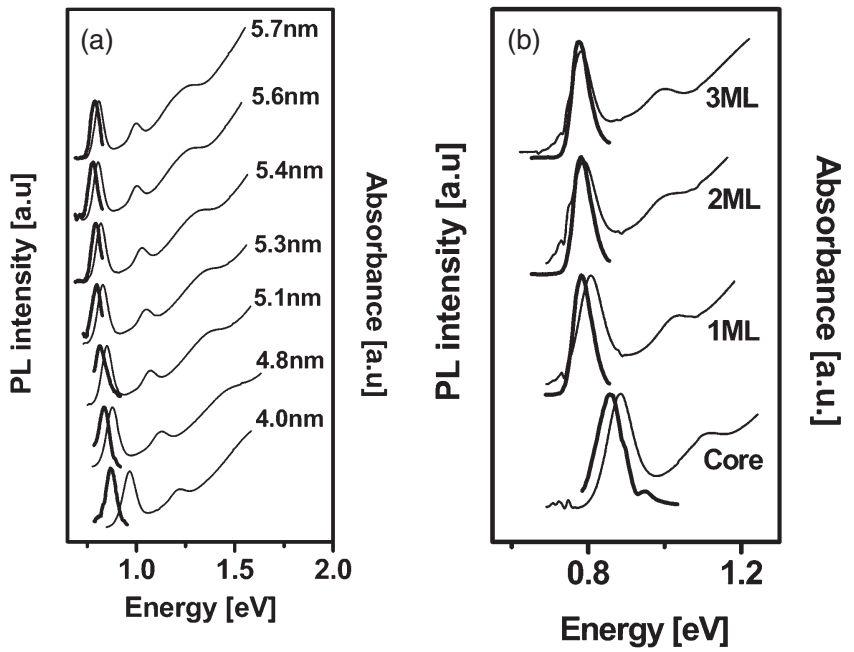

Figure 9.20 (a) Absorbance (thin lines) and corresponding PL (bold lines) spectra of core PbSe nanocrystals with NC diameters as indicated; (b) Absorbance (thin lines) and corresponding PL spectra (bold lines) of $4.9 \mathrm{~nm}$ PbSe core (lowest curves) and the corresponding $\mathrm{PbSe} / \mathrm{PbS}$ core-shell (top three curves) nanocrystals with $n$ monolayers $(n=1,2$ and 3) of a PbS shell. Reproduced from Ref. [76].

of 2.3 to $7.0 \mathrm{~nm}$ was demonstrated; spectral evidence was also provided for a decrease in the Stokes shift with increasing particle size (Figure 9.20).

The preparation of $\mathrm{PbSe} / \mathrm{PbSe}_{x} \mathrm{~S}_{1-x}$ core-shells structures could be achieved by using a single injection of the precursors into a single round flask. For this, a stock solution of Se, S was prepared by mixing $0.15 \mathrm{~g}$ Se dissolved in $1.4 \mathrm{ml} \mathrm{TOP}$, with $0.03-0.10 \mathrm{~g} \mathrm{~S}$ dissolved in $0.3 \mathrm{ml}$ trioctylphosphine. The amount of $\mathrm{S}$ in the new stock solution corresponded to a stoichiometric amount of one to two monolayers of the $\mathrm{PbS}$ compound. Thus, the molar ratio of the precursors $\mathrm{Pb}: \mathrm{Se}: \mathrm{S}$ ranged between 10:9:5 and 7:6:14. The periodic removal of aliquots during the reaction was followed by purification and examination of the species by HR-TEM and absorption spectroscopy. The results suggested a rapid nucleation of the $\mathrm{PbSe}$ core, followed by a slower precipitation of the $\mathrm{PbSe}_{x} \mathrm{~S}_{1-x}$ shell, with a gradual change in the chemical composition. This single process is reported to produce materials of a narrow size distribution (5\%) and high quantum efficiencies (55\%) when compared to conventional core and core-shell materials. The claim was also made that these systems, $\mathrm{PbSe} / \mathrm{PbS}$ and $\mathrm{PbSe} / \mathrm{PbSe} \mathrm{S}_{x} \mathrm{~S}_{1-x}$ core-shell nanocrystal QDs, were air-stable and therefore possessed the advantage that no further processing would be required to protect the surfaces from atmospheric oxidation for applications [73]. However, in studies conducted by Stouwdam et al., who investigated the photostability of colloidal $\mathrm{PbSe}$ and $\mathrm{PbSe} / \mathrm{PbS}$ core-shell nanocrystals in solution and in the solid state, no differences were observed in stability between the coated NCs as compared to uncoated NCs in solution upon irradiation with a 
xenon lamp [72]. The possible reason given for this was that either the $\mathrm{PbS}$ shell was unable to confine the charge carriers, or there was incomplete shell passivation within the $\mathrm{PbSe}$ core and, as a result, the core-shell NCs had comparable stability. These findings highlighted and typified a number of problems encountered when attempting to characterize the structure and stability of these systems. PbSe has also been coated with Group II-VI semiconductors to controllably synthesize $\mathrm{PbSe} / \mathrm{CdSe}$ type-I core-shell QDs that are stable against fading and spectral shifting [69]. These core-shells can then further undergo additional shell growth to produce $\mathrm{PbSe} / \mathrm{CdSe} / \mathrm{ZnS}$ CSS QDs that represent the initial steps toward bright, biocompatible, NIR optical labels.

The coating of $\mathrm{PbTe}$ has been carried out using controlled oxidation in the presence of amines [77], and by cation exchange to form $\mathrm{PbTe} / \mathrm{CdTe}$. In the former case the oleylamine induces the formation of $\mathrm{Pb}(\mathrm{OH})_{2}$ or lead oxide at the surface of the PbTe nanocrystals. If no treatment is involved to remove the excessive oleylamine from the surfaces of the freshly prepared PbTe after PbTe NC synthesis, the $\mathrm{Te}^{2-}$ in $\mathrm{PbTe}$ nanocrystals can be partially replaced by $\mathrm{OH}^{-}$from the trace amount of water present. This reaction is facilitated by the presence of oleylamine, and initially produces a shell of amorphous $\mathrm{Pb}(\mathrm{OH})_{2}$. The shell may subsequently be transformed into crystalline $\mathrm{PbO}$ after an annealing treatment, while retaining the particle shape. The material retains its narrow size distribution and forms supercrystals - this is an interesting observation, given that the nanoparticles are cubes and not spheres. Hence, through an anion-exchange mechanism in the presence of oleylamine, the $\mathrm{PbTe}$ nanocubes can be converted into core-shell building blocks by "shrinking" themselves into a truncated octahedral or a near-spherical core with an amorphous but quasi-cubic shell (PbTe@ $\mathrm{Pb}(\mathrm{OH})_{2}$ and PbTe@PbO). The cubic core-shell particles can still be packed into a twodimensional pattern or a super crystal, although the long-range order was lost due to a slight truncation of the corners of the NCs ( Figure 9.21).

In a cation-exchange reaction, Lambert et al. took the $\mathrm{PbS} / \mathrm{CdS}$ and $\mathrm{PbSe} / \mathrm{CdSe}$ core-shell formation by cation exchange (as reported by Pietryga and coworkers) as a starting point and extended it to $\mathrm{PbTe} / \mathrm{CdTe}$. They also showed that a combination of the PbTe rocksalt structure and the CdTe zinc blende structure allowed for direct observation of the core and shell with HRTEM. This enabled a direct visualization of the crystallographic properties of the PbTe/CdTe QDs, and an evaluation of the cation-exchange reaction. A seamless match was also observed between the PbTe and CdTe crystal lattices. It was concluded that, in the case of lead chalcogenides, cationic exchange represented a straightforward technique for the formation of lead chalcogenide/cadmium chalcogenide core-shell materials.

\section{7}

\section{Summary and Conclusions}

In this chapter, progress in the synthesis of colloidal nanoheterostructures was summarized. Depending on their electronic structure (type-I or type-II) and their 

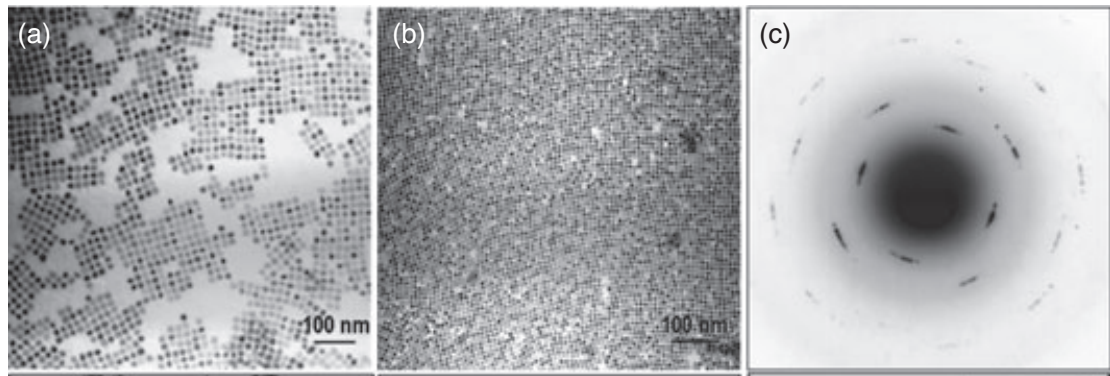

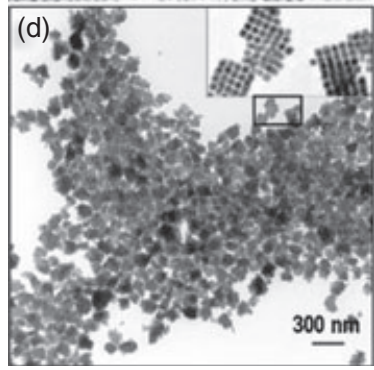

(e)

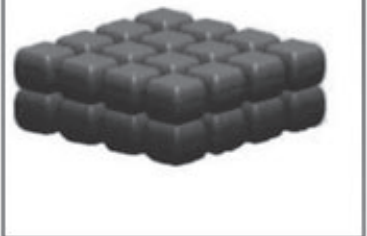

(f)

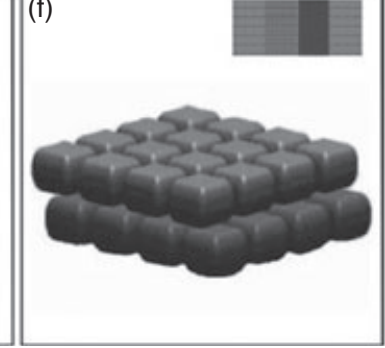

Figure 9.21 Electron microscopy images of PbTe nanocubes and simple cubic (SC).

(a) TEM image of PbTe monolayer assemblies; (b) TEM image of PbTe monolayer assembly; (c) Selected area electron diffraction pattern of monolayer assembly (negative pattern); (d) TEM image of PbTe multilayer assemblies; (e) Model of stacking structure of two-layer assembly ("simple cubic SC"); ( $f$ ) Model of stacking structure of two-layer assembly ("1D-shifted SC"). Reproduced from Ref. [77].

geometry (spherical or nonspherical), the properties and application potential of these structures was discussed. Type-I structures were introduced as potent dyes with very high fluorescence quantum yields and high photostability, while type-II heterostructures may be used for extended band gap engineering. The latter continue to show promising properties with regards to amplified stimulated emission.

\section{References}

1 Spanhel, L., Haase, M., Weller, H. and Henglein, A. (1987) Photochemistry of colloidal semiconductors. 20. Surface modification and stability of strong luminescing CdS particles. Journal of the American Chemical Society, 109, 5649-55.

2 Murray, C.B., Norris, D.J. and Bawendi, M.G. (1993) Synthesis and characterization of nearly monodisperse $\mathrm{CdE}$ (E = sulfur, selenium, tellurium) semiconductor nanocrystallites. Journal of the American Chemical Society, 115, 8706-15.
3 Hines, M.A. and Guyot-Sionnest, P. (1996) Synthesis and characterization of strongly luminescing ZnS-Capped CdSe nanocrystals. Journal of Physical Chemistry, 100, 468-71.

4 Dabbousi, B.O. et al. (1997) (CdSe)ZnS core-shell quantum dots: synthesis and characterization of a size series of highly luminescent nanocrystallites. Journal of Physical Chemistry B, 101, 9463-75.

5 Peng, X.G., Schlamp, M.C., Kadavanich, A.V. and Alivisatos, A.P. (1997) Epitaxial growth of highly luminescent CdSe/CdS 
core/shell nanocrystals with photostability and electronic accessibility. Journal of the American Chemical Society, 119, 7019-29.

6 Li, J.J. et al. (2003) Large-scale synthesis of nearly monodisperse CdSe/CdS core/ shell nanocrystals using air-stable reagents via successive ion layer adsorption and reaction. Journal of the American Chemical Society, 125, 12567-75.

7 Steckel, J.S. et al. (2004) Blue luminescence from (CdS)ZnS core-shell nanocrystals. Angewandte Chemie, International Edition, 43, 2154-8.

8 Haubold, S., Haase, M., Kornowski, A. and Weller, H. (2001) Strongly luminescent InP/ZnS core-shell nanoparticles. ChemPhysChem, 2, 331-4.

9 Bruns, O.T. et al. (2009) Real-time magnetic resonance imaging and quantification of lipoprotein metabolism in vivo using nanocrystals. Nature. Nanotechnology, 4, 193-201.

10 Kim, S., Fisher, B., Eisler, H.J. and Bawendi, M. (2003) Type-II quantum dots: $\mathrm{CdTe} / \mathrm{CdSe}$ (core/shell) and CdSe/ $\mathrm{ZnTe}$ (core/shell) heterostructures. Journal of the American Chemical Society, 125, 11466-7.

11 Ivanov, S.A. et al. (2007) Type-II core/ shell CdS/ZnSe nanocrystals: synthesis, electronic structures, and spectroscopic properties. Journal of the American Chemical Society, 129, 11708-19.

12 Klimov, V.I. et al. (2007) Single-exciton optical gain in semiconductor nanocrystals. Nature, 447, 441-6.

13 Eychmüller, A., Mews, A. and Weller, H. (1993) A quantum-dot quantumwell-CdS/HgS/CdS. Chemical Physics Letters, 208, 59-62.

14 Mews, A., Eychmüller, A., Giersig, M., Schooss, D. and Weller, H. (1994) Preparation, characterization, and photophysics of the quantum-dot quantum-well system CdS/HgS/CdS. Journal of Physical Chemistry, 98, 934-41.

15 Schooss, D., Mews, A., Eychmüller, A. and Weller, H. (1994) Quantum-dot quantum-well CdS/HgS/CdS - theory and Experiment. Physical Review B, 49, 17072-8.

16 Bryant, G.W. (1995) Theory for quantumdot quantum wells: pair correlation and internal quantum confinement in nanoheterostructures. Physical Review $B$, 52, 16997-7000.

17 Jaskolski, W. and Bryant, G.W. (1998)

Multiband theory of quantum-dot quantum wells: dim excitons, bright excitons, and charge separation in heteronanostructures. Physical Review B, 57, R4237-40.

18 Eychmüller, A., Vossmeyer, T., Mews, A. and Weller, H. (1994) Transient photobleaching in the quantum-dot quantum-well CdS/HgS/CdS. Journal of Luminescence, 58, 223-6.

19 Kamalov, V.F., Little, R., Logunov, S.L. and El-Sayed, M.A. (1996) Picosecond electronic relaxation in $\mathrm{CdS} / \mathrm{HgS} / \mathrm{CdS}$ quantum dot quantum well semiconductor nanoparticles. Journal of Physical Chemistry, 100, 6381-4.

20 Banin, U., Mews, A., Kadavanich, A.V., Guzelian, A.A. and Alivisatos, A.P. (1996) Homogeneous optical properties of semiconductor nanocrystals. Molecular Crystals and Liquid Crystals Science and Technology. Section A: Molecular Crystals and Liquid Crystals, 283, 1-10.

21 Mews, A., Kadavanich, A.V., Banin, U. and Alivisatos, A.P. (1996) Structural and spectroscopic investigations of $\mathrm{CdS} / \mathrm{HgS} /$ CdS quantum-dot quantum wells. Physical Review B, 53, 13242-5.

22 Lifshitz, E. et al. (1999) Optically detected magnetic resonance study of CdS/HgS/ CdS quantum dot quantum wells. Journal of Physical Chemistry, B, 103, 6870-5.

23 Little, R.B., El-Sayed, M.A., Bryant, G.W and Burke, S. (2001) Formation of quantum-dot quantum-well heteronanostructures with large lattice mismatch: ZnS/CdS/ZnS. Journal of Chemical Physics, 114, 1813-22.

24 Battaglia, D., Li, J.J., Wang, Y.J. and Peng, X.G. (2003) Colloidal two-dimensional systems: CdSe quantum shells and wells. Angewandte Chemie, International Edition, 42, 5035-9.

25 Dorfs, D. and Eychmüller, A. (2001) A series of double well semiconductor quantum dots. Nano Letters, 1, 6635.

26 Braun, M., Burda, C. and El-Sayed, M.A (2001) Variation of the thickness and number of wells in the CdS/HgS/CdS quantum dot quantum well system. 
Journal of Physical Chemistry, A, 105, 5548-51.

27 Borchert, H. et al. (2003) Photoemission study of onion like quantum dot quantum well and double quantum well nanocrystals of CdS and HgS. Journal of Physical Chemistry, B, 107, 7486-91.

28 Dorfs, D., Henschel, H., Kolny, J. and Eychmüller, A. (2004) Multilayered nanoheterostructures: theory and experiment. Journal of Physical Chemistry, $B, 108,1578-83$.

29 Reiss, P., Carayon, S., Bleuse, J. and Pron, A. (2003) Low polydispersity core/ shell nanocrystals of CdSe/ZnSe and $\mathrm{CdSe} / \mathrm{ZnSe} / \mathrm{ZnS}$ type: preparation and optical studies. Synthetic Metals, 139, 649-52.

30 Talapin, D.V. et al. (2004) CdSe/CdS/ZnS and $\mathrm{CdSe} / \mathrm{ZnSe} / \mathrm{ZnS}$ core-shell-shell nanocrystals. Journal of Physical Chemistry, $B, 108,18826-31$.

31 Jun, S., Jang, E. and Lim, J.E. (2006) Synthesis of multi-shell nanocrystals by a single step coating process. Nanotechnology, 17, 3892-6.

32 Xie, R.G., Kolb, U., Li, J.X., Basche, T. and Mews, A. (2005) Synthesis and characterization of highly luminescent CdSe-Core CdS/Zn0.5Cd0.5S/ZnS multishell nanocrystals. Journal of the American Chemical Society, 127, 7480-8.

33 Aharoni, A., Mokari, T., Popov, I. and Banin, U. (2006) Synthesis of InAs/CdSe/ ZnSe core/shell1/shell2 structures with bright and stable near-infrared fluorescence. Journal of the American Chemical Society, 128, 257-64.

34 Battaglia, D., Blackman, B. and Peng, X.G. (2005) Coupled and decoupled dual quantum systems in one semiconductor nanocrystal. Journal of the American Chemical Society, 127, 10889-97.

35 Dias, E.A., Sewall, S.L. and Kambhampati, P. (2007) Light harvesting and carrier transport in core/barrier/shell semiconductor nanocrystals. Journal of Physical Chemistry, C, 111, 708-13.

36 Sapra, S., Mayilo, S., Klar, T.A., Rogach, A.L. and Feldmann, J. (2007) Bright white-light emission from semiconductor nanocrystals: by chance and by design. Advanced Materials, 19, 569-72.

37 Peng, X.G. et al. (2000) Shape control of CdSe nanocrystals. Nature, 404, 59-61.
38 Carbone, L. et al. (2007) Synthesis and micrometer-scale assembly of colloidal $\mathrm{CdSe} / \mathrm{CdS}$ nanorods prepared by a seeded growth approach. Nano Letters, 7 , 2942-50.

39 Milliron, D.J. et al. (2004) Colloidal nanocrystal heterostructures with linear and branched topology. Nature, 430 , 190-5.

40 Talapin, D.V. et al. (2003) Highly emissive colloidal CdSe/CdS heterostructures of mixed dimensionality. Nano Letters, 3, 1677-81.

41 Kraus, R.M. et al. (2007) Roomtemperature exciton storage in elongated semiconductor nanocrystals. Physical Review Letters, 98, 017401-1-017401-4.

42 Dorfs, D., Salant, A., Popov, I. and Banin, U. (2008) ZnSe quantum dots within CdS nanorods: a seeded-growth type-II system. Small, 4, 1319-23.

43 Fiore, A. et al. (2009) Tetrapod-shaped colloidal nanocrystals of II-VI semiconductors prepared by seeded growth. Journal of the American Chemical Society, 131, 2272-82.

44 Borchert, H. et al. (2003) High resolution photoemission study of CdSe and CdSe/ ZnS core-shell nanocrystals. Journal of Chemical Physics, 119, 1800-7.

45 Rogach, A.L., Eychmüller, A., Hickey, S.G. and Kershaw, S.V. (2007) Infraredemitting colloidal nanocrystals: synthesis, assembly, spectroscopy, and applications. Small, 3, 536-57.

46 Chen, C.Y. et al. (2005) Type-II CdSe/ $\mathrm{CdTe} / \mathrm{ZnTe}$ (core-shell-shell) quantum dots with cascade band edges: the separation of electron (at CdSe) and hole (at ZnTe) by the CdTe layer. Small, 1, 1215-20.

47 Peng, P. et al. (2005) Femtosecond spectroscopy of carrier relaxation dynamics in type-II CdSe/CdTe tetrapod heteronanostructures. Nano Letters, $\mathbf{5}$, 1809-13.

48 Wang, J., Long, Y.T., Zhang, Y.L., Zhong, X.H. and Zhu, L.Y. (2009) Preparation of highly luminescent CdTe/CdS core/shell quantum dots. ChemPhysChem, 10, 680-5.

49 Kim, S. et al. (2009) Bandgap engineered reverse type-I CdTe/InP/ZnS core-shell nanocrystals for the near-infrared. Chemical Communications, 1267-9. 
50 Kairdolf, B.A., Smith, A.M. and Nie, S. (2008) One-pot synthesis, encapsulation, and solubilization of size-tuned quantum dots with amphiphilic multidentate ligands. Journal of the American Chemical Society, 130, 12866-7.

51 Xia, Y.S. and Zhu, C.Q. (2008) Aqueous synthesis of type-II core/shell CdTe/CdSe quantum dots for near-infrared fluorescent sensing of copper(II). Analyst, 133, 928-32.

52 Seo, H. and Kim, S.W. (2007) In situ synthesis of CdTe/CdSe core-shell quantum dots. Chemistry of Materials, 19, 2715-17.

53 Chou, P.T. et al. (2006) Spectroscopy and femtosecond dynamics of type-II CdTe/ CdSe core-shell quantum dots. ChemPhysChem, 7, 222-8.

54 Tsay, J.M., Pflughoefft, M., Bentolila, L.A. and Weiss, S. (2004) Hybrid approach to the synthesis of highly luminescent $\mathrm{CdTe} / \mathrm{ZnS}$ and CdHgTe/ZnS nanocrystals. Journal of the American Chemical Society, 126, 1926-7.

55 Lifshitz, E. and Eychmuller, A. (2007) Spectroscopic investigations on II-VIsemiconductor nanocrystals and their assemblies. Journal of Cluster Science, 18, 5-18.

56 Harrison, M.T. et al. (2000) Wet chemical synthesis of highly luminescent HgTe/ CdS core/shell nanocrystals. Advanced Materials, 12, 123-5.

$57 \mathrm{Xu}$, S., Ziegler, J. and Nann, T. (2008) Rapid synthesis of highly luminescent InP and InP/ZnS nanocrystals. Journal of Materials Chemistry, 18, 2653-6.

58 Langof, L. et al. (2004) Colloidal InP/ZnS core-shell nanocrystals studied by linearly and circularly polarized photoluminescence. Chemical Physics, 297, 93-8.

59 Cao, Y. and Banin, U. (2000) Growth and properties of semiconductor core/shell nanocrystals with InAs cores. Journal of the American Chemical Society, 122, 9692-702.

60 Ben-Lulu, M., Mocatta, D., Bonn, M., Banin, U. and Ruhman, S. (2008) On the absence of detectable carrier multiplication in a transient absorption study of InAs/CdSe/ZnSe core/shell1/ shell2 quantum dots. Nano Letters, 8 , 1207-11.
61 Zimmer, J.P. et al. (2006) Size series of small indium arsenide-zinc selenide core-shell nanocrystals and their application to in vivo imaging. Journal of the American Chemical Society, 128, 2526-7.

62 Millo, O., Katz, D., Cao, Y. and Banin, U. (2001) Tunneling and optical spectroscopy of InAs and InAs/ZnSe core/shell nanocrystalline quantum dots. Physica Status Solidi, (B) Basic Research, 224, 271-6.

63 Kim, S.W. et al. (2005) Engineering In $\mathrm{As}_{\mathrm{x}} \mathrm{P}_{1 \cdot \mathrm{x}} / \mathrm{InP} / \mathrm{ZnSe}$ III-V alloyed core/ shell quantum dots for the near-infrared. Journal of the American Chemical Society, 127, 10526-32.

64 Hua, B., Motohisa, J., Kobayashi, Y., Hara, S. and Fukui, T. (2009) Single GaAs/GaAsP coaxial core-shell nanowire lasers. Nano Letters, 9, 112-16.

65 Prete, P. et al. (2008) Luminescence of $\mathrm{GaAs} / \mathrm{AlGaAs}$ core-shell nanowires grown by MOVPE using tertiary butylarsine. Journal of Crystal Growth, 310, 5114-18.

66 Titova, L.V. et al. (2006) Temperature dependence of photoluminescence from single core-shell GaAs-AlGaAs nanowires. Applied Physics Letters, 89, 2865-7.

67 Noborisaka, J., Motohisa, J., Hara, S. and Fukui, T. (2005) Fabrication and characterization of freestanding GaAs/ AlGaAs core-shell nanowires and AlGaAs nanotubes by using selective-area metalorganic vapor phase epitaxy. Applied Physics Letters, 87, 093109-1-093109-3.

68 Koktysh, D.S., McBride, J.R., Dixit, S.K., Feldman, L.C. and Rosenthal, S.J. (2007) $\mathrm{PbS} / \mathrm{PbSe}$ structures with core-shell type morphology synthesized from $\mathrm{PbS}$ nanocrystals. Nanotechnology, 18 , 495607-1-495607-4.

69 Pietryga, J.M. et al. (2008) Utilizing the lability of lead selenide to produce heterostructured nanocrystals with bright, stable infrared emission. Journal of the American Chemical Society, 130, 487985.

70 Cui, D., Xu, J., Paradee, G., Xu, S.Y. and Wang, A.Y. (2007) Developing PbSe/PbS core-shell nanocrystals quantum dots toward their potential heterojunction applications. Journal of Experimental Nanoscience, 2, 13-21. 
$71 \mathrm{Xu}$, J. et al. (2006) Synthesis and surface modification of $\mathrm{PbSe} / \mathrm{PbS}$ core-shell nanocrystals for potential device applications. Nanotechnology, 17, 542834.

72 Stouwdam, J.W. et al. (2007) Photostability of colloidal $\mathrm{PbSe}$ and $\mathrm{PbSe} /$ $\mathrm{PbS}$ core/shell nanocrystals in solution and in the solid state. Journal of Physical Chemistry, C, 111, 1086-92.

73 Lifshitz, E. et al. (2006) Air-stable PbSe/ $\mathrm{PbS}$ and $\mathrm{PbSe} / \mathrm{PbSe}_{\mathrm{x}} \mathrm{S}_{1-\mathrm{x}}$ core-shell nanocrystal quantum dots and their applications. Journal of Physical Chemistry, $B, 110,25356-65$.

74 Brumer, M. et al. (2006) Nanocrystals of $\mathrm{PbSe}$ core, $\mathrm{PbSe} / \mathrm{PbS}$, and $\mathrm{PbSe} / \mathrm{PbSe}_{x} \mathrm{~S}_{1-\mathrm{x}}$ core/shell as saturable absorbers in passively Q-switched near-infrared lasers. Applied Optics, 45, 7488-97.

75 Kigel, A., Brumer, M., Sashchiuk, A., Amirav, L. and Lifshitz, E. (2005) PbSe/ $\mathrm{PbSe}_{\mathrm{x}} \mathrm{S}_{1-\mathrm{x}}$ core-alloyed shell nanocrystals. Materials Science \& Engineering C-Biomimetic and Supramolecular Systems, 25, 604-8.

76 Brumer, M. et al. (2005) PbSe/PbS and $\mathrm{PbSe} / \mathrm{PbSe} \mathrm{S}_{1-\mathrm{x}}$ core/shell nanocrystals. Advanced Functional Materials, 15, 1111-16.

77 Zhang, J. et al. (2008) Simple cubic super crystals containing PbTe nanocubes and their core-shell building blocks. Journal of the American Chemical Society, 130, 15203-9.

78 Lambert, K., De Geyter, B., Moreels, I. and Hens, Z. (2009) PbTe/CdTe core/ shell particles by cation exchange: A HRTEM study. Chemistry of Materials, 21, 778-80.

79 Lim, M.A., Il Seok, S., Chung, W.J. and Hong, S.I. (2008) Near infrared luminescence properties of nanohybrid film prepared from $\mathrm{LaPO}_{4}: \mathrm{Er}^{3+} / \mathrm{LaPO}_{4}$ core/shell nanoparticles and silica-based resin. Optical Materials, 31, 201-5.

80 Zhang, M.F. et al. (2008) Preparation and characterization of near-infrared luminescent bifunctional core/shell nanocomposites. Journal of Physical Chemistry, C, 112, 2825-30.
81 Lee, J.S., Shevchenko, E.V. and Talapin, D.V. (2008) Au-PbS core-shell nanocrystals: plasmonic absorption enhancement and electrical doping via intra-particle charge transfer. Journal of the American Chemical Society, 130, 9673-5.

82 Schwartzberg, A.M., Grant, C.D., van Buuren, T. and Zhang, J.Z. (2007) Reduction of $\mathrm{HAuCl}_{4}$ by $\mathrm{Na}_{2} \mathrm{~S}$ revisited: the case for $\mathrm{Au}$ nanoparticle aggregates and against $\mathrm{Au}_{2} \mathrm{~S} / \mathrm{Au}$ Core/Shell particles. Journal of Physical Chemistry, C, 111, 8892-901.

83 Norman, T.J. et al. (2002) Near infrared optical absorption of gold nanoparticle aggregates. Journal of Physical Chemistry, $B, 106,7005-12$.

84 Paltiel, Y., Aharoni, A., Banin, U., Neuman, O. and Naaman, R. (2006) Self-assembling of InAs nanocrystals on GaAs: the effect of electronic coupling and embedded gold nanoparticles on the photoluminescence. Applied Physics Letters, 89, 033108-1-033108-3.

85 Xuan, Y. et al. (2007) Near infrared light-emitting diodes based on composites of near infrared dye, CdSe/CdS quantum dots and polymer. Semiconductor Science and Technology, 22, 1021-4.

86 Dubertret, B. et al. (2002) In vivo imaging of quantum dots encapsulated in phospholipid micelles. Science, 298, 1759-62.

87 Ablyazov, N.N., Areshkin, A.G., Melekhin, V.G., Suslina, L.G. and Fedorov, D.L. (1986) Fluctuation-induced broadening of exciton reflection spectra in $\mathrm{A}^{\mathrm{II}} \mathrm{B}^{\mathrm{VI}}$ solid-solutions. Physica Status Solidi B-Basic Research, 135, 217-25.

88 Niles, D.W. and Hochst, H. (1990) Band offsets and interfacial properties of cubic CdS grown by molecular-beam epitaxy on CdTe(110). Physical Review B, 41, 12710-19.

89 Hines, M.A. and Scholes, G.D. (2003) Colloidal PbS nanocrystals with sizetunable near-infrared emission: observation of post-synthesis selfnarrowing of the particle size distribution. Advanced Materials, 15, 1844-9. 